\title{
A Mathematical Study of a Generalized SEIR Model of COVID-19
}

\author{
Abdelkader Intissar ${ }^{\text {a, }}$ b* $^{*}$ \\ ${ }^{a}$ Equipe d'Analyse Spectrale, UMR-CNRS n: 6134, Université de Corse, Quartier Grossetti, 20250 Corté, France. \\ ${ }^{b}$ Le Prador, 129 rue du commandant Rolland, 13008 Marseille, France.
}

Received 06 August 2020; Accepted 27 August 2020

\begin{abstract}
In this work (Part I), we reinvestigate the study of the stability of the Covid-19 mathematical model constructed by Shah et al. (2020) [1]. In their paper, the transmission of the virus under different control strategies is modeled thanks to a generalized SEIR model. This model is characterized by a five dimensional nonlinear dynamical system, where the basic reproduction number $R_{0}$ can be established by using the next generation matrix method. In this work (Part I), it is established that the disease free equilibrium point is locally as well as globally asymptotically stable when $R_{0}<1$. When $R_{0}>1$, the local and global asymptotic stability of the equilibrium are determined employing the second additive compound matrix approach and the Li-Wang's (1998) stability criterion for real matrices [2]. In the second paper (Part II), some control parameters with uncertainties will be added to stabilize the five-dimensional Covid-19 system studied here, in order to force the trajectories to go to the equilibria. The stability of the Covid-19 system with these new parameters will also be assessed in Intissar (2020) [3] applying the Li-Wang criterion and compound matrices theory. All sophisticated technical calculations including those in part I will be provided in appendices of the part II.
\end{abstract}

Keywords: Epidemic Models; Endemic Equilibrium; Stability of Matrices; Next Generation Matrix; Second Additive Compound Matrix; Global Stability; Dynamical Systems; Covid-19 Model.

\section{Introduction}

The evolution of epidemics is one of the most dangerous problems for a society. As mankind already faced severe pandemics such as the Spanish flu in 1917, the Honk Kong flu (H3N2) in 1968 and the swine flu (H1N1) in 2009, the forecast of epidemics evolution appears to be one of the most critical topics for our societies. On January 7, 2020, the isolation of a new coronavirus by a team of Chinese scientists, causing severe acute respiratory syndrome for the patients infected with this virus [4,5] (later designated coronavirus disease 2019 (Covid-19) by the World Health Organization), shed a new light on this issue.

Several efforts were done since the 1970's in order to understand the spread of diseases and to forecast their evolution through mathematical models. Amongst the various papers and preprints published to better understand the properties of the Covid-19 and model its evolution in different countries, a mathematical Covid-19 model was constructed by Shah et al. (2020) [1] to study the human to human transmission of the Covid-19. This model is reinvestigated in this paper.

The work is organized as follows:

- In section 1, the mathematical Covid-19 model and its parameters are presented, alongside with some preliminary results on linear stability analysis for systems of ordinary differential equations.

* Corresponding author: intissar@univ-corse.fr; abdelkader.intissar@orange.fr

doi http://dx.doi.org/10.28991/SciMedJ-2020-02-SI-4

$>$ This is an open access article under the CC-BY license (https://creativecommons.org/licenses/by/4.0/).

(C) Authors retain all copyrights. 
- In section 2, the Li-Wang's stability criterion for real matrices (used to study the stability of an epidemic model of SEIR type with varying total population) is presented and some spectral properties of M-matrices are recalled.

- In section 3, some preliminary definitions and some lemmas for linear stability of our (covid-19) system are provided.

- To that regard, a very important threshold quantity is the basic reproduction number, sometimes called the basic reproductive number or basic reproductive ratio (Heffernan et al. 2005 [6]), which is usually denoted by $R_{0}$.

From an epidemiological perspective, $R_{0}$ refers to the average number of secondary cases produced by one infected individual introduced into a population of susceptible individuals, where an infected individual has acquired the disease, and susceptible individuals are healthy but can acquire the disease. In reality, the value of $R_{0}$ for a specific disease depends on many variables, such as location and density of population.

- In section 4 , the study of the stability of equilibrium points of the (Covid-19) system is performed using the $R_{0}$ criterion and Li-Wang criterion on second additive compound matrix associated to Jacobian matrix of the (covid19) system.

The study of the stability of Jacobian matrices of an order less than three of a dynamic system yields a reasonable $R_{0}$, but for more complex compartmental models, especially those with more infected compartments, the study of the stability is difficult as it relies on the algebraic Routh-Hurwitz conditions for stability of the Jacobian matrix. An alternative method proposed by Diekmann et al. (1990) [7] and elaborated by van den Driessche and Watmough (2002) [8] gives a way of determining $R_{0}$ for a compartmental model by using the next generation matrix.

The main part of the section 4 is the determination of equilibrium points of our Covid-19 system and the explicit calculation of additive compound matrix of Jacobian matrix associated to this system. In this work, it is the first time that the explicit calculation of a second additive compound matrix associated with a square matrix of order 5 is given.

\section{Presentation of Mathematical Covid-19 Model With Its Parameters and Some Preliminary Results}

A mathematical Covid-19 model is constructed by Shah et al. (2020) [1] to study human to human transmission of the Covid-19.

The model consists all possible human to human transmission of the virus.

The Covid-2019 is highly contagious in nature and infected cases are seen in most of the countries around the world, hence in the model the susceptible population class is ignored and whole population is divided in five compartments:

(1) class of exposed individuals $\mathrm{E}(\mathrm{t})$ (individuals surrounded by infection by not yet infected),

(2) class of infected individuals by Covid-19 I(t),

(3) class of critically infected individuals by Covid-19 C(t),

(4) class of hospitalised individuals $\mathrm{H}(\mathrm{t})$, and

(5) class of dead individuals due to Covid-19 D(t).

Human to human transmission dynamics of Covid-19 is describe graphically in

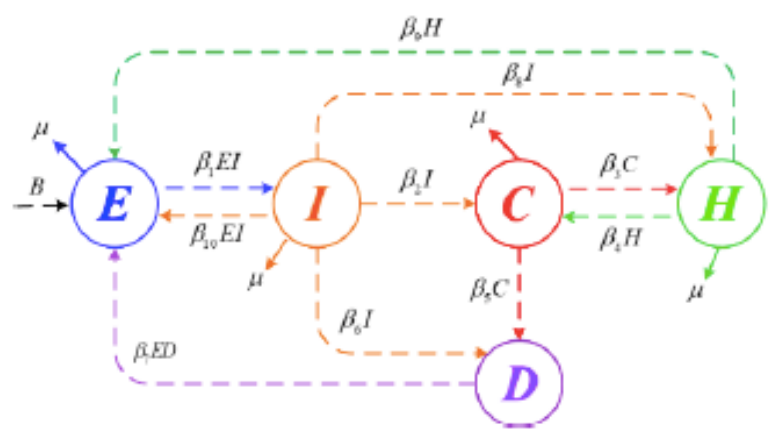

- Table of parameters used in the model is described as follow:

B : Birth rate of class of exposed individuals : 0.80 Calculated

$\mu$ Natural death rate : 0.01 Assumed 
$\beta_{1}$ : Transmission rate of individuals moving from exposed to infected class: 0.55 Calculated

$\beta_{2}$ : Rate at which infected individuals goes into sever condition or in critical condition : 0.40 Calculated

$\beta_{3}$ : Rate at which critically infected individuals get hospitalized :0.60 Calculated

$\beta_{4}$ : Rate by which hospitalized individuals not recovered and remain in critical condition: 0.80 Calculated

$\beta_{5}$ : Mortality rate of critically infected individuals: 0.34 Calculated

$\beta_{6}$ : Mortality rate of infected individuals: 0.30 Calculated

$\beta_{7}$ : Rate by which infected dead body spreads infection: 0.35 Assumed

$\beta_{8}$ : Rate at which infected individuals get hospitalized: 0.30 Calculated

$\beta_{9}$ : Rate at which hospitalised individuals get recovered and become exposed again : 0.35 Assumed

$\beta_{10}$ : Rate at which infected individuals recovered themselves due to strong immunity and again become exposed

Using the above representation, dynamical system of set of nonlinear differential for the model is formulated as follow:

$\left\{\begin{array}{l}\frac{d E}{d t}=B-\beta_{1} E I+\beta_{7} E D+\beta_{9} H+\beta_{10} E I-\mu E \\ \frac{d I}{d t}=\beta_{1} E I-\beta_{2} I-\beta_{6} I-\beta_{8} I-\beta_{10} E I-\mu I \\ \frac{d C}{d t}=\beta_{2} I-\beta_{5} C-\beta_{3} C+\beta_{4} H-\mu C \\ \frac{d H}{d t}=\beta_{3} C-\beta_{4} H+\beta_{8} I-\beta_{9} H-\mu H \\ \frac{d D}{d t}=\beta_{5} C+\beta_{6} I-\beta_{7} D E\end{array}\right.$

(Covid-19)

\section{Remark 1.1}

(i) All of the parameters in (covid-19) are assumed to be nonnegative.

(ii) $\frac{d E}{d t}+\frac{d I}{d t}+\frac{d C}{d t}+\frac{d H}{d t}+\frac{d D}{d t}=B-\mu(E+I+C+H+D)$

(iii) $\frac{d E}{d t}+\frac{d I}{d t}+\frac{d C}{d t}+\frac{d H}{d t}+\frac{d D}{d t} \leq 0 \Leftarrow E+I+C+H+D \leq \frac{B}{\mu}$

(iv) A detailed description of the model can be found in shah et al. (2020) [1] and there in references.:

(v) For other mathematical systems of epidemic models, we can consult these references [9-11].

\section{Theorem 1.2}

(i) The positive orthant $\mathbb{R}_{+}^{5}$ is positively invariant under the flow of (covid-19). Precisely, if $E(0)>0 ; I(0)>0$; $C(0)>0 ; H(0)>0 ; D(0)>0$ then $\forall t>0 ; E(t)>0 ; I(t)>0 C(t)>0 ; H(t)>0 ; D(t)>0$.

\section{Proof}

(i) - Let's suppose $I(0)>0$, then from the second equation of (covid-19)), if

$\chi(t)=\left(\beta_{10}-\beta_{1}\right) E+\beta_{2}+\beta_{6}+\beta_{8}+\mu$ then the integration from 0 to $t>0$ gives:

$I(t)=I(0) e^{-\int_{0}^{t} \chi(s) d s}$.

Therefore $I(t)>0 ; \forall t \geq 0$.

- Consider the following sub-equations related to the variables $C$ and $H$ :

$\left\{\begin{array}{l}\frac{d C(t)}{d t}=\beta_{2} I(t)-\beta_{5} C(t)-\beta_{3} C(t)+\beta_{4} H(t)-\mu C(t) \\ \frac{d H(t)}{d t}=\beta_{3} C(t)-\beta_{4} H(t)+\beta_{8} I(t)-\beta_{9} H(t)-\mu H(t) \\ C(0)>0 \text { and } H(0)>0\end{array}\right.$ 
The system (1.3) takes the matrix form:

$\frac{d U}{d t}=M U(t)+F(t)$

where

$M=\left(\begin{array}{cc}-\left(\beta_{5}+\beta_{3}+\mu\right) & \beta_{4} \\ \beta_{3} & -\left(\beta_{4}+\beta_{9}+\mu\right)\end{array}\right)$

And;

$U(t)=\left(\begin{array}{l}C(t) \\ H(t)\end{array}\right) ; \quad U(0)=\left(\begin{array}{l}C(0) \\ H(0)\end{array}\right)>0$ and $F(t)=\left(\begin{array}{l}\beta_{2} I(t) \\ \beta_{8} I(t)\end{array}\right)>0$.

One can turn the Cauchy problem (1.3) into an integral equation by using the following so called Duhamel formula:

$U(t)=e^{t M} U(0)+\int_{0}^{t} e^{(t-s) M} F(s) d s$

Therefore $C(t)>0 ; \forall t \geq 0$. and $H(t)>0 ; \forall t \geq 0$.

We can observe also that $M$ is a Metzler matrix (a matrix $A=\left(a_{i j} 1 \leq i, j \leq n\right.$ is a Metzler matrix if all of its elements are non-negative except for those on the main diagonal, which are unconstrained.) That is, a Metzler matrix is any matrix $A$ which satisfies $A=\left(a_{i j}\right) ; \quad a_{i j} \geq 0, \quad i \neq j$.

Thus, (1.3) is a monotone system. It follows that, $\mathbb{R}_{+}^{2}$ is invariant under the flow of (1.3).

- Let's suppose $E(0)>0$, then from the first equation of (covid-19)), if

$\chi(t)=\left(\beta_{1}-\beta_{10}\right) I-\beta_{7} D+\mu$ and $\pi(t)=\beta_{9} H(t)+B$ which is $>0$.

the integration from 0 to $t>0$ gives:

$E(t)=E(0) e^{-\int_{0}^{t} \chi(s) d s}+e^{-\int_{0}^{t} \chi(s) d s} \int_{0}^{t} \pi(u) e^{\int_{0}^{u} \chi(w) d w} d u$.

Therefore $E(t)>0 ; \forall t \geq 0$.

- Let's suppose $D(0)>0$, then from the $5^{\text {th }}$ equation of (covid-19)), if

$\chi(t)=\beta_{7} E$ and $\pi(t)=\beta_{6} I(t)+\beta_{5} C(t)$ which is $>0$.

the integration from 0 to $t>0$ gives :

$D(t)=D(0) e^{-\int_{0}^{t} \chi(s) d s}+e^{-\int_{0}^{t} \chi(s) d s} \int_{0}^{t} \pi(u) e^{\int_{0}^{u} \chi(w) d w} d u$.

Therefore $D(t)>0 ; \forall t \geq 0$.

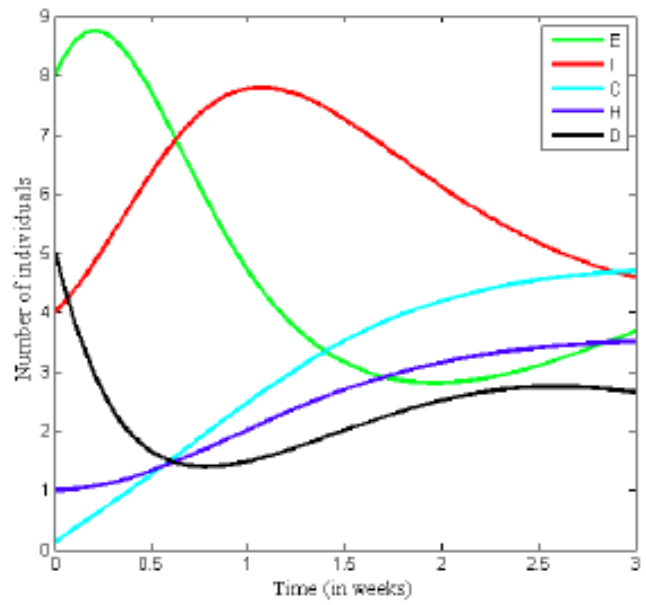

\section{Remark 1.3}

(i) The theorem 1.2 ensures the existence and uniqueness of global (in time) solution of system (covid-19).

(ii) Let $\Lambda$ be the domain $\Lambda=\left\{(E, B, C, H, D) \in \mathbb{R}_{+}^{5} ; E+I+C+H+D \leq \frac{B}{\mu}\right\}$ then this domain is positively invariant, and all the solutions of the system (covid-19) are remain in this domain. 
Consider the following n-dimensional system:

$x^{\prime}(t)=f(x(t)) ; t \geq 0$

where $f: \Omega \subset \mathbb{R}^{n} \rightarrow \mathbb{R}^{n}$ is $\mathcal{C}^{1}$-function.

\section{Definition 1.4}

- We say that $x^{*}$ is an equilibrium point of (1.6) if $f\left(x^{*}\right)=0$.

- We will say that an equilibrium point $x^{*}$ is stable if:

$\forall \epsilon>0, \exists \delta>0$ such that $\left\|x-x^{*}\right\|<\delta$ and $t>0 \Rightarrow\left\|\phi_{t}(x)-x^{*}\right\|<\epsilon$

where $\phi_{t}(x)$ is a solution of (1.6)

- We will say that an equilibrium point $x^{*}$ is asymptotically stable if for each neighborhood $\mathbb{U}$ of $x^{*}$ there exists a neighborhood $\mathbb{W}$ such that $x^{*} \in \mathbb{W} \subset \mathbb{U}$ and $x(0) \in \mathbb{W}$ implies that the solution $\phi_{t}(x)$ satisfies $\phi_{t}(x) \in \mathbb{U}$ for all $t>0$, and that $\phi_{t}(x) \rightarrow x^{*}$ as $t \rightarrow+\infty$.

In particular, a system is called asymptotically stable around its equilibrium point at the origin if it satisfies the following two conditions:

1. Given any $\epsilon>0 ; \exists \delta_{1}>0$ such that if $\|x(0)\|<\delta_{1}$, then

$\left\|\phi_{t}(x)\right\|<\epsilon, \forall t>0$.

2. $\exists \delta_{1}>0$ such that if $\|x(0)\|<\delta_{2}$, then $\phi_{t}(x) \rightarrow 0$ as $t \rightarrow \infty$.

The first condition requires that the state trajectory can be confined to an arbitrarily small "ball" centered at the equilibrium point and of radius $\epsilon$, when released from an arbitrary initial condition in a ball of sufficiently small (but positive) radius $\delta_{1}$. This is called stability in the sense of Lyapunov (i.s.L.).

It is possible to have stability in the sense of Lyapunov without having asymptotic stability, in which case we refer to the equilibrium point as marginally stable. Nonlinear systems also exist that satisfy the second requirement without being stable i.s.L. An equilibrium point that is not stable i.s.L. is termed unstable.

\section{- Linear stability analysis for systems of ordinary differential equations}

Consider the n-dimensional dynamical system (1.6) written in the following form:

$\frac{d x_{i}}{d t}=f_{i}(x(t))$;

$x(t)=\left(x_{1}(t), \ldots, x_{i}(t), \ldots, x_{n}(t), 1 \leq i \leq n\right.$ and $0 \leq t<+\infty$

Where:

$x(0)=\left(x_{1}(0), \ldots, x_{i}(0), \ldots, x_{n}(0)\right)=x_{0}$ is fixed

And;

$f_{i}: \mathbb{R}^{n} \rightarrow \mathbb{R}$ are $\mathcal{C}^{1}$-functions which are given.

and suppose that $x^{*}=\left(x_{1}^{*}, \ldots, x_{i}^{*}, \ldots, x_{n}^{*}\right)$ is a steady state, that is, $f_{i}\left(x^{*}\right)=0$.

The question of interest is whether the steady state is stable or unstable. Consider a small perturbation from the steady state by letting $x_{i}=x_{i}^{*}+u_{i}, 1 \leq i \leq n$ where both $u_{i}, 1 \leq i$ are understood to be small. The question of interest translates into the following: will $u_{i}, 1 \leq i$ where both grow (so that $x_{i}, 1 \leq i \leq n$ move away from the steady state), or will they decay to zero (so that $x_{i}, 1 \leq i \leq n$ move towards the steady state)?

In the former case, we say that the steady state is unstable, in the latter it is stable.To see whether the perturbation grows or decays, we need to derive differential equations for $u_{i}, 1 \leq i$ We do so as follows:

$\frac{d u_{i}}{d t}=\frac{d x_{i}}{d t}, 1 \leq i \leq n$ (since $x_{i}^{*}$ is constant $1 \leq i \leq n$ )

$=f_{i}(x)$ (by definition)

$=f_{i}\left(x^{*}+u\right), u=\left(u_{1}, \ldots, u_{i}, \ldots . u_{n}\right)$ (substitution)

$=f_{i}\left(x^{*}\right)+\sum_{j=1}^{n} \frac{\partial f_{i}}{\partial x_{j}}\left(x^{*}\right)+\ldots$. (Taylor series expansion)

$=\sum_{j=1}^{n} \frac{\partial f_{i}}{\partial x_{j}}\left(x^{*}\right)+\ldots .\left(\right.$ since $\left.f_{i}\left(x^{*}\right)=0\right)$

The .... denote higher order terms, Since $u_{i} ; 1 \leq i \leq n$ are assumed to be small, these higher order terms are 
extremely small.

The above linear system for $u_{i} ; 1 \leq i \leq n$ has the trivial steady state $u_{i}=0 ; 1 \leq i \leq n$, and the stability of this trivial steady state is determined by the eigenvalues of the matrix, as follows:

If we can safely neglect the higher order terms, we obtain the following linear system of equations governing the evolution of the perturbations $u_{i}, 1 \leq i \leq n$ :

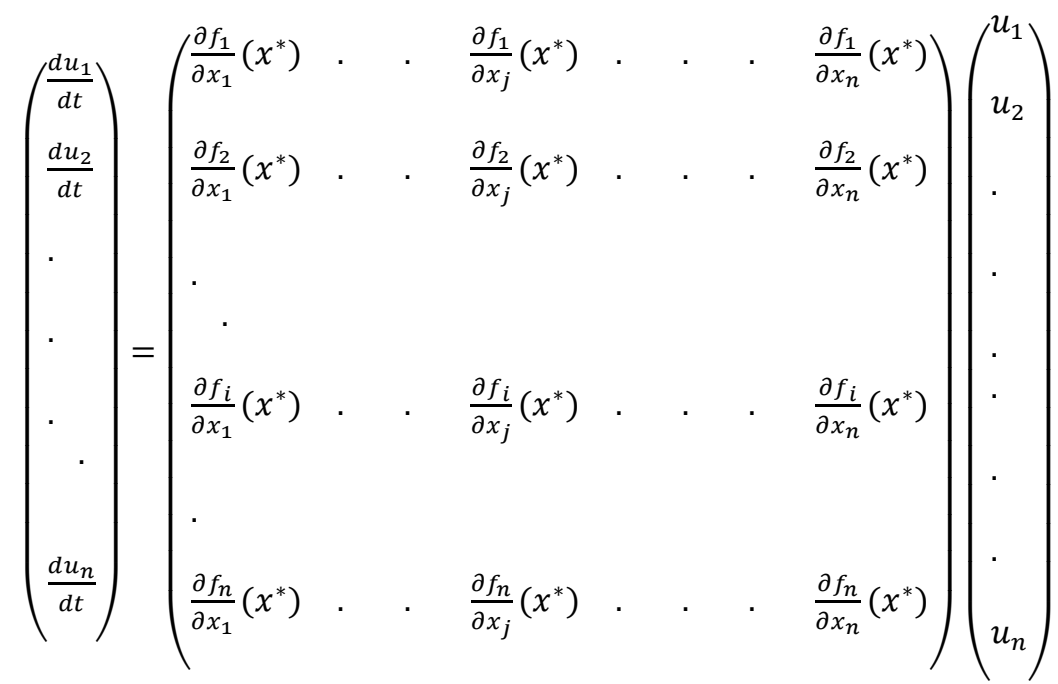

We refer to the matrix as the Jacobian matrix of the original system at the steady state $x^{*}$.

$\frac{d u}{d t}=\mathbb{J}_{x^{*}} u$ where $\mathbb{J}_{x^{*}}=\left(\frac{\partial f_{i}\left(x^{*}\right)}{\partial x_{j}}\right)_{1 \leq i, j \leq n}$

\section{Theorem 1.5}

if the eigenvalues of the Jacobian matrix all have real parts less than zero, then the steady state is stable.

if the eigenvalues of the Jacobian matrix all have real parts $<0$, then the steady state is asymptotically stable.

If at least one of the eigenvalues of the Jacobian matrix has real part greater than zero, then the steady state is unstable.

Otherwise there is no conclusion (then we have a borderline case between stability and instability; such cases require an investigation of the higher order terms we neglected, and this requires more sophisticated mathematical machinery discussed in advanced courses on ordinary differential equations).

\section{Definition 1.6}

An equilibrium point $x^{*}$ is said hyperbolic if all eigenvalues of the Jacobian matrix have real parts $\neq 0$.

\section{Remark 1.7}

A hyperbolic equilibrium point $x^{*}$ is asymptotically stable if the eigenvalues of the Jacobian matrix all have real parts $<0$ or otherwise it is unstable.

Let $A$ be the Jacobian matrix, assume that it is a real hyperbolic matrix, i.e. $\Re e \lambda \neq 0$ for for all eigenvalues $\lambda$ of $A$, then

There is a linear change of variables [good coordinates $\left(x_{s}, x_{u}\right)$ ] that induces a splitting into stable and unstable spaces $\mathbb{R}^{n}=\mathcal{E}_{s} \oplus \mathcal{E}_{u}$ so that in the new variables

$$
A=\left(\begin{array}{ll}
A_{s} & 0 \\
0 & A_{u}
\end{array}\right)
$$

and a constant $\alpha>0$ so that for $t \geq 0$,

$$
\left\{\begin{array}{l}
\left\|e^{t A} x_{s}\right\| \leq e^{-\alpha t}\left\|x_{s}\right\| \\
\left\|e^{-t A} x_{u}\right\| \leq e^{-\alpha t}\left\|x_{u}\right\|
\end{array}\right.
$$


We have written $x_{s}=P_{s} x, x_{u}=P_{u} x$ where $P_{s}: \mathbb{R}^{n} \rightarrow \mathcal{E}_{s}$ and $P_{u}: \mathbb{R}^{n} \rightarrow \mathcal{E}_{u}$ are the orthogonal projections.

Last but not least, there is a theorem (the Hartman- Grobman Theorem) that guarantees that the stability of the steady state $x^{*}$ of the original system is the same as the stability of the trivial steady state 0 of the linearized system.

Let $x^{*}$ be an equilibrium point of nonlinear system (1.6) then by applying a translation, we can always assume 0 is a equilibrium point of (1.6).

- Poincaré in his dissertation showed that if $f$ is analytic at the equilibrium point $x^{*}$, and the eigenvalues of $\mathbb{J}_{x^{*}}$ are nonresonant, then there is a formal power series of change of variable to change (1.6) to a linear system [4, 12] .

- Hartman and Grobman showed that if $f$ is continuously differentiable, then there is a neighborhood of a hyperbolic equilibrium point and a homeomorphism on this neighborhood, such that the system in this neighborhood is changed to a linear system under such a homeomorphism [13-17].

\section{Theorem 1.8 (Hartman-Grobman theorem)}

Let $\Omega$ be an open set of $\mathbb{R}^{n}$ containing the origin, $f: \Omega \rightarrow \mathbb{R}^{n}$ be a $\mathcal{C}^{1}$ - function on $\Omega, 0$ be a hyperbolic equilibrium point of the system (1.6), and $\mathbb{U}_{r}=\{x ;\|x\|<r\}$ be the neighborhood of the origin of radius $r$. For any $r, \epsilon>0$ such that $\overline{\mathbb{U}}_{r+\epsilon} \subset \Omega$, there exists a transformation $y=H(x), H(0)=0$ and $H$ is a homeomorphism in a neighborhood of 0 , such that the system (1.6) is changed into the linear system

$$
y^{\prime}(t)=\mathbb{A} y, \quad \mathbb{A}=\left(\frac{\partial f_{i}(0)}{\partial x_{j}}\right)_{1 \leq i, j \leq n} \text { in } \mathbb{U}_{r}
$$

Proof

See: http://www.math.utah.edu/ treiberg/M6414HartmanGrobman.pdf.

Thus, the procedure to determine stability of $x^{*}$ is as follows:

1. Compute all partial derivatives of the right-hand-side of the original system of differential equations, and construct the Jacobian matrix.

2. Evaluate the Jacobian matrix at the steady state.

3. Compute eigenvalues.

4. Conclude stability or instability based on the real parts of the eigenvalues.

Definition 1.9 (Liapunov function)

Let $x^{*}$ be an equilibrium point of (1.6), $\mathbb{U} \subset \Omega$ be a neighborhood of $x^{*}$ and $L: \mathbb{U} \rightarrow \mathbb{R}$ be a continuous function. We say that $L$ is Liapunov function for (1.6) at $x^{*}$ if

(1) $L\left(x^{*}\right)=0$ and for every $x \neq x^{*}$ we have $L(x)>0$;

(2) The function $t \rightarrow L\left(\phi_{t}(x)\right)$ is decreasing.

We say that $L$ is strictly Liapunov function for (1.6) at $x^{*}$ if $L$ satisfy (1) and

(3) the function $t \rightarrow L\left(\phi_{t}(x)\right)$ is strictly decreasing.

\section{Remark 1.10}

If $L$ is $\mathcal{C}^{1}$ function then we can replace :

- The condition (2) by $\forall x \in \mathbb{U},<\nabla L(x), f(x)>\leq 0$.

and

- The condition (3) by $\forall x \in \mathbb{U},<\nabla L(x), f(x)><0$.

\section{Theorem 1.11}

If (1.6) admits a Liapunov function at an equilibrium point $x^{*}$, then $x^{*}$ is stable and if the Liapunov function is strictly decreasing then $x^{*}$ is asymptotically stable.

We outline in the next section the Li-Wang's stability criterion for real matrices and we recall of some spectral properties of $M-$ matrices. 


\section{On Li-Wang's Stability Criterion of Real Matrix}

\section{Definition 2.1}

Let $\mathbb{A}$ be an $n \times n$ matrix and let $\sigma(\mathbb{A})$ be its spectrum. The stability modulus of $\mathbb{A}$ is defined by $s(\mathbb{A})=$ $\operatorname{Max}\{\mathcal{R e} \lambda ; \lambda \in \sigma(\mathbb{A})\}$ i.e. s(A) is the maximum real part of the eigenvalues of $\mathbb{A}$ called also the spectral abscissa.

$\mathbb{A}$ is said to be stable if $s(\mathbb{A})<0$.

The stability of a matrix is related to the Routh-Hurwitz problem on the number of zeros of a polynomial that have negative real parts. Routh-Hurwitz discovered necessary and sufficient conditions for all of the zeros to have negative real parts, which are known today as the Routh-Hurwitz conditions. A good and concise account of the Routh-Hurwitz problem can be found in Banks et al. (1992) [5].

The Li-Wang criterion offer an alternative to the well-known Routh-Hurwitz. It based on Lozinski $\breve{\imath}$ measures and second additive compound matrix. For detailed discussions on compound matrices, the reader is referred to Li-Wang Li-Wang [2] and for additive compound matrices to Fiedler (1974) [18].

- In Li-Wang [2] a necessary and sufficient condition for the stability of an $n \times n$ matrix with real entries is derived (Li-Wang criterion) by using a simple spectral property of additive compound matrices.

- A survey is given of a connection between compound matrices and ordinary differential equations by Muldowney (1990) [19].

And for an application of Li-Wang criterion, we can consult [7, 20-22].

Now, let $\mathbb{M}_{n}(\mathbb{K})$ be the linear space of $n \times n$ matrices with entries in $\mathbb{K}$,

where $\mathbb{K}=\mathbb{R}$ or $\mathbb{C}$.

\section{Definition 2.2}

- Let $\wedge$ denote the exterior product in $\mathbb{K}^{n}$, and let $1 \leq k \leq n$ be an integer. With respect to the canonical basis in the $k t h$ exterior product space $\wedge^{k} \mathbb{K}^{n}$, the $k t h$ additive compound matrix $\mathbb{A}{ }^{[k]}$ of $\mathbb{A}$ is a linear operator on $\wedge^{k} \mathbb{K}^{n}$ whose definition on a decomposable element $x_{1} \wedge x_{2} \wedge \ldots \ldots \wedge x_{k}$ is

$$
\mathbb{A}^{[k]} x_{1} \wedge x_{2} \wedge \ldots \ldots \wedge x_{k}=\sum_{i=1}^{k} x_{1} \wedge x_{2} \wedge \ldots \wedge \mathbb{A} x_{i} \wedge \ldots \wedge x_{k}
$$

- Let $\mathbb{A}=\left(a_{i j}\right)_{1 \leq i, j \leq n}$ and for any integer $i=1, \ldots, C_{n}^{k}$, let $((i))=\left(i_{1}, i_{2}, \ldots, i_{k}\right)$ be the ith member in the lexicographic ordering of integer $k$-tuples such that $1 \leq i_{1}<i_{2}<\ldots<i_{1} \leq n$ where $C_{n}^{k}=\frac{n !}{k !(n-k) !}$. Then

- The entry in the ith row and the $j$ th column of $\mathbb{A}^{[k]}=\left(\hat{a}_{i j}\right)_{1 \leq i, j \leq C_{n}^{k}}$ is

$$
\hat{a}_{i, j}=\left\{\begin{array}{l}
a_{i_{1}, i_{1}}+\ldots \ldots a_{i_{k}, i_{k}} \text { if }((i))=((j)) ; 1 \leq i_{1}<i_{2} \leq n \\
(-1)^{r+s} a_{j_{r}, i_{s}} \text { if exactly one entry of } i_{s} \text { does not } \\
\text { occur in }((j)) \text { and } j_{r} \text { does not occur in }((i)), \\
0 \text { if }((i)) \text { differs from }((j)) \text { in two or more entries. }
\end{array}\right.
$$

- Let $\|$.$\| denote a vector norm in \mathbb{K}^{n}$ and the operator norm it induces in $\mathbb{M}_{n}(\mathbb{K})$.

${ }_{1}$ The Lozinskiı̆ measure $\mu$ (also known as logarithmic norm $\|.\|_{\text {log }}$ ) on $\mathbb{M}_{n}(\mathbb{K})$ with respect to $\|$.$\| is defined by$ (see Coppel (1965) [23])

For $\mathbb{A} \in \mathbb{M}_{n}(\mathbb{K})$,

$\mu(A):{ }_{h \rightarrow 0^{+}} \operatorname{Lim} \frac{\|\mathbb{I}+h \mathbb{A}\|-1}{h}$

$\circ_{2}$ By the logarithmic norm of a matrix $A$ we mean the real number defined by the formula :

$\|A\|_{\log }:={ }_{t \rightarrow 0^{+}} \operatorname{Lim} \frac{\ln \|\mathbb{I}+h \mathbb{A}\|-\|I\|}{t}$

- The existence of a limit in (2.3) $b i s$ is established on the basis of the convexity of the function $I+t A$ (see Bylov (1996) [24], Supplement I, Sec. 2), whence we also borrow the notation for the logarithmic norm). 
- The logarithmic norm of a matrix for an arbitrary norm was introduced by the Leningrad mathematician Lozinskii (1958) [25] and the Swedish mathematician Dahlquist (1959) [26] in their papers on the numerical integration of ordinary differential equations. For linear bounded operators in Banach spaces, a similar notion was introduced Daletskii and Krein (1970) [27], Problems and supplement to Chap. I.

- Let $A=\left(a_{i j}\right)$ be a real or complex square $n \times n$ matrix, and let $\lambda_{1}, \lambda_{2}, \ldots, \lambda_{n}$ be the complete set of its eigenvalues denoted by $\sigma(A)$ (the spectrum of the matrix $A$ ). The maximal real part of these eigenvalues is denoted by $s(A)$ i.e. $s(A)=\max _{1 \leq i \leq n} \Re e \lambda_{i}$. (spectral abscissa).

The term "spectral abscissa" (by analogy with the spectral radius $\rho(A)=\lim \left\|A^{n}\right\|^{\frac{1}{n}}$ as $n \rightarrow+\infty$ of a matrix $A$ ) and the notation for it were proposed in Perov (2002) [28].

- What the best upper and lower bounds for $\left\|e^{t A}\right\| ?, 0 \leq t<+\infty$ where $e^{t A}=I+\sum_{k=1}^{n} \frac{t^{k} A^{k}}{k !}$.

It follows from the definition of $e^{t A}$ that $e^{-t\|A\|} \leq\left\|e^{t A}\right\| \leq e^{t\|A\|}, 0 \leq t<+\infty$ but $-\|A\|$ and $\|A\|$ are not the best constants.

Now, let $\alpha$ and $\beta$ the best constants in the estimate :

$e^{t \alpha} \leq\left\|e^{t A}\right\| \leq e^{\beta t}, 0 \leq t<+\infty$

the existence of such constants is beyond doubt.

\section{Theorem 2.3}

(i) Let $\alpha$ be the best constant in estimate (2.4) from below. Then

$$
\alpha=i n f_{0<t} \frac{\ln \left(\left\|e^{t A}\right\|\right)}{t}=\lim \frac{\ln \left(\left\|e^{t A}\right\|\right)}{t}=\max _{1 \leq i \leq n} \mathfrak{R} e \lambda_{i} \text { as } t \rightarrow+\infty \text {. }
$$

(ii) Let $\beta$ be the best constant in estimate (2.4) from below. Then

$$
\beta=\sup _{0<t} \frac{\ln \left(\left\|e^{t A}\right\|\right)}{t}=\lim \frac{\ln \|I+t A\|}{t}=\lim \frac{\ln \|I+t A\| \mid-\|I\|}{t} \text { as } t \rightarrow 0^{+} .
$$

\section{Proof}

(i) For the proof, see (Daletskii and Krein (1970) [27], Chap. I, Theorem 4.1).

We see from the last equality in (2.5) that $\alpha$ is the spectral abscissa of the matrix $A: \alpha=s(A)$. Let us stress that the spectral abscissa is independent of the choice of the norm.

(ii) We see from the last equality in (2.6) that $\beta$ is the logarithmic norm of the matrix $A: \beta=\|A\|_{\log }$.

Consider the logarithmic function on the positive semi-axis. In view of its continuous differentiability, it locally satisfies the Lipschitz condition. Therefore, for any $\epsilon>0$, we can indicate a $\delta=\delta_{\epsilon}>0$ such that

$|\ln u-\ln v| \leq(1+\epsilon)|u-v|$ for $|u-1|<\delta,|v-1|<\delta$.

Therefore, under the conditions $\left\|\left|e^{t A} \|-1\right|<\delta\right.$ and $\||I+t A \|-1|<\delta$, we have

$|\ln ||\exp (t A)\|-\ln \| I+t A\|\leq(1+\epsilon)\||\left|e^{t A}\|-\| I+t A \|\right|$

$\| e^{t A}-\left(I+t A\left\|\leq \sum_{k=2}^{n} \frac{t^{k}\|A\|^{k}}{k !}=e^{t|| A \|}-1-t\right\| A \|\right.$.

Therefore,

$$
\lim _{t \rightarrow 0^{+}} \frac{\ln \left\|e^{t A}\right\|}{t}=\lim _{t \rightarrow 0^{+}} \frac{\ln \|I+t A\|}{t},
$$

Provided that at least one of the limits in (2.7) exists.

Further, setting $\epsilon(t)=\|I+t A\|-\|I\|$, we can write

$$
\begin{cases}\frac{\ln \|I+t A\|}{t}=\frac{\|I+t A\|-\|I\|}{t} & \text { if } \epsilon(t)=0 \\ \frac{\ln || I+t A \|}{t}=\frac{\ln (1+\epsilon(t))}{\epsilon(t)} \frac{\|I+t A\| \mid-\|I\|}{t} & \text { if } \epsilon(t) \neq 0\end{cases}
$$

whence, using the well-known relation $\frac{\ln (1+x)}{x} \rightarrow 1$ as $x \rightarrow 0$, we obtain

$\lim _{t \rightarrow 0^{+}} \frac{\ln \|I+t A\|}{t}=\lim _{t \rightarrow 0^{+}} \frac{\|I+t A\||-| \mid I \|}{t}$ 
Provided that at least one of the limits in (2.8) exists. As we have already said above, the last limit exists and serves to define the logarithmic norm. It remains to prove that

$$
\sup _{0<t} \frac{\ln \left\|e^{t A}\right\|}{t}=\lim _{t \rightarrow 0^{+}} \frac{\ln \left\|e^{t A}\right\|}{t}
$$

In (2.9), the quantity on the left exists, is finite and is equal to $\beta$; as proved above, the limit on the right exists, is finite and will be denoted by $b$. The definition of the number $\beta$ implies the inequality $\beta \geq b$.

Suppose for the time being that the written inequality is strict: $\beta>b$. For a sufficiently small $\epsilon>0$, we can write $\beta-\epsilon \geq b+\epsilon$. From the obtained $\epsilon>0$, we then find a $\delta=\delta_{\epsilon}$ such that

$\left\|e^{t A}\right\| \leq\left\|e^{t(b+\epsilon)}\right\|$ for $0<t \leq \delta$.

After this, consider an arbitrary fixed $t>0$. Let us choose a natural number $k$ so that $0<\frac{t}{k} \leq \delta$.

After this, we estimate

$$
\left\|e^{t A}\right\|=\left\|e^{k \frac{t}{k} A}\right\| \leq\left\|e^{\frac{t}{k} A}\right\|^{k} \leq e^{\frac{t}{k}(b+\epsilon) k}=e^{t(b+\epsilon)} \leq e^{t(\beta-\epsilon)} .
$$

Thus,

$\left\|e^{t A}\right\| \leq e^{t(\beta-\epsilon)}$ for $0<t<+\infty$,

and this explicitly contradicts the definition of the number $\beta$.

This Theorem implies the important inequality:

$\alpha=s(A) \leq\|A\|_{\log }=\beta$.

For every $A, B \in M_{n}(\mathbb{C}), \alpha \geq 0$, and $\xi \in \mathbb{C}$ the following relations hold:

• $1 \mu(\alpha A+\xi I)=\alpha \mu(A)+\mathfrak{R} e \xi$.

$\cdot_{2}-\|A\| \leq-\mu(-A) \leq \mu(A) \leq\|A\|$.

-3 $\mu(A)+\mu(-A) \geq 0$

- $_{4} \mu(A+B) \leq \mu(A)+\mu(B)$.

$\bullet_{5}-\mu(-A) \leq \Re e \lambda \leq \mu(A)$ for $\lambda \in \sigma(A)$.

In the partial case for the Holder vector $p$-norm defined by

$\|x\|_{p}=\left(\sum_{i=1}^{n}\left|x_{i}\right|^{p}\right)^{\frac{1}{p}}$ and $\|x\|_{\infty}=\max _{1 \leq i \leq n}\left\{\left|x_{i}\right|\right\}$

then the corresponding matrix measure can be calculated explicitly in the cases:

\section{- Example 1}

- (a) Let $n \in \mathbb{N}, X=\left(x_{1}, \ldots, x_{i}, \ldots, x_{n}\right) \in \mathbb{R}^{n}$ with vector norm $\|X\|=\sum_{i=1}^{n}\left|x_{i}\right|$ and

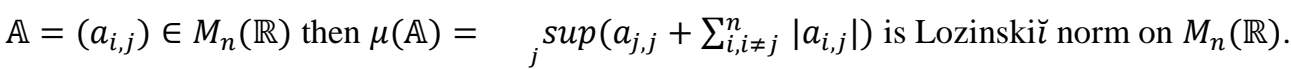

- (b) The Lozinski measures of complex matrix $\mathbb{A}=\left(a_{i, j}\right) \in M_{n}(\mathbb{C})$ with respect to the three common norms $\|x\|_{\infty}=\sup _{i}\left|x_{i}\right|,\|x\|_{1}=\sum_{i=1}^{n}\left|x_{i}\right|$ and $\|x\|_{2}=\sqrt{\sum_{i}^{n}\left|x_{i}\right|^{2}}$ are

$\mu_{\infty}(A)=\sup _{i}\left(\mathfrak{R e a} a_{i i}+\sum_{k, k \neq i}\left|a_{i k}\right|, \mu_{1}(A)=\sup _{k}\left(\mathfrak{R e} a_{k k}+\sum_{i, i \neq k}\left|a_{i k}\right|\right.\right.$ and $\mu_{2}(A)=s\left(\frac{A+A^{*}}{2}\right)$ respectively, where $A^{*}$ denotes the Hermitian adjoint of $A$.

If $A$ is real symmetric, then $\mu_{2}(A)=s(A)$.

For a real matrix A, conditions $\mu_{\infty}(A)<0$ or $\mu_{1}(A)<0$ can be interpreted as $a_{i i}<0$

for $i=1, \ldots, n$, and $A$ is diagonally dominant in rows or in columns, respectively.

- Some upper and lower bounds for the determinant of $\boldsymbol{n} \times \boldsymbol{n}$ matrix $\boldsymbol{A}$ with positive diagonal elements

Let $A=\left(a_{i j}\right)_{1 \leq i, j \leq n}$ be a real matrix satisfying:

$a_{i i} \geq \sum_{j \neq i}\left|a_{i j}\right|, \quad i=1,2, \ldots, n$

Then we have the following result: 


\section{Theorem 2.4}

If $A=\left(a_{i j}\right)_{1 \leq i, j \leq n}$ has elements satisfying (2.4), it is possible to define $l_{i}$ and $r_{i}$, such that :

$\left\{\begin{array}{l}a_{i i}=l_{i}+r_{i}, 1 \leq i \leq n \\ l_{i} \geq \sum_{j<i}\left|a_{i j}\right|, 1 \leq i \leq n \\ r_{i} \geq \sum_{j>i}\left|a_{i j}\right|, 1 \leq i \leq n\end{array}\right.$

Then, for any choice of $l_{i}$ and $r_{i}$, satisfying (2.5) we have

$\sum_{k=0}^{n}\left(\prod_{i=1}^{k} l_{i} \prod_{i=k+1}^{n} r_{i}\right) \leq \operatorname{det} A \leq \sum_{k=0}^{n}\left(\prod_{i=1}^{k-1}\left(l_{i}+2 r_{i}\right) l_{i} \prod_{i=k+1}^{n} r_{i}\right)$

where an empty product is defined to be 1 and $\operatorname{det} A$ denotes determinant of $A$.

\section{Proof}

To prove this result, we need the following bound given by Price (1951) [29]:

If (2.4) holds then

$\prod_{i=1}^{n}\left(a_{i i}-r_{i}\right) \leq \operatorname{det} A \leq \prod_{i=1}^{n}\left(a_{i i}+r_{i}\right)$

where $r_{i}=\sum_{j>i}\left|a_{i j}\right|$.

Let $\mathbb{D}_{n}$ represent the determinant of $A$, then we proceed by induction on $n$ :

(a) For $n=2$, let $A=\left(\begin{array}{ll}a_{11} & a_{12} \\ a_{21} & a_{22}\end{array}\right)$ has elements satisfying (2.4) then $r_{1}=\left|a_{12}\right|$ and $r_{2}=0$ then by observing that :

$\cdot_{1}\left|a_{12}\right| a_{22} \geq\left|a_{12}\right|\left|a_{21}\right|=\left|a_{12} a_{21}\right| \geq-a_{12} a_{21}$.

and

- $_{2}\left|a_{12}\right| a_{22} \geq\left|a_{12}\right|\left|a_{21}\right|=\left|a_{12} a_{21}\right| \geq a_{12} a_{21}$.

We deduce that the Price's theorem holds.

Now let $\mathbb{D}_{2}=\left|\begin{array}{ll}a_{11} & a_{12} \\ a_{21} & a_{22}\end{array}\right|=\left|\begin{array}{ll}l_{1}+r_{1} & a_{12} \\ a_{21} & l_{2}+r_{2}\end{array}\right|$ and expanding it by diagonal elements in the following form:

$\mathbb{D}_{2}=\left|\begin{array}{ll}l_{1} & a_{12} \\ 0 & l_{2}\end{array}\right|+\left|\begin{array}{ll}l_{1} & 0 \\ 0 & r_{2}\end{array}\right|+\left|\begin{array}{ll}r_{1} & 0 \\ a_{21} & r_{2}\end{array}\right|+\left|\begin{array}{ll}r_{1} & a_{12} \\ a_{21} & l_{2}\end{array}\right|$.

Therefore:

$l_{1} l_{2}+l_{1} r_{2}+r_{1} r_{2} \leq \mathbb{D}_{2} \leq r_{1} r_{2}+l_{1} r_{2}+\left(l_{1}+2 r_{1}\right) l_{2}$,

Since

$0 \leq\left|\begin{array}{ll}r_{1} & a_{12} \\ a_{21} & l_{2}\end{array}\right| \leq\left(r_{1}+a_{12}\right) l_{2}<2 r_{1} l_{2}$ by $(\mathbf{2 . 5})$ and $(\mathbf{2 . 7})$.

(b) Assume that for any matrix of order $n-1$ with elements satisfying (2.5),

$\sum_{k=0}^{n-1}\left(\prod_{i=1}^{k} l_{i} \prod_{i=k+1}^{n_{1}} r_{i}\right) \leq \mathbb{D}_{n-1} \leq \sum_{k=0}^{n-1}\left(\prod_{i=1}^{k-1}\left(l_{i}+2 r_{i}\right) l_{i} \prod_{i=k+1}^{n-1} r_{i}\right)$

If $\mathbb{D}_{n}=\operatorname{det} A$, where $A=\left(a_{i j}\right) 1 \leq i, j \leq n$, and the elements $a_{i j}$ satisfy (2.5), partition $\mathbb{D}_{n}$ as follows :

$\mathbb{D}_{n}=\left|\begin{array}{ll}A_{1} & \hat{a}_{2} \\ \hat{a}_{3} & l_{n}+r_{n}\end{array}\right|$. 
Where:

$A_{1}=\left(a_{i j}\right) 1 \leq i, j \leq n-1, \hat{a}_{2}$ is the column vector with components $a_{i n} 1 \leq i \leq n-1, \hat{a}_{3}$ is the row vector with components $a_{n j} 1 \leq j \leq n-1$, and as in (2.5), $l_{n}+r_{n}=a_{n n}, l_{n} \geq \sum_{j=1}^{n-1} \mid a_{n j}, r_{n} \geq 0$.

Then we can write $\mathbb{D}_{n}$ as the sum of two determinants, i.e.,

$\mathbb{D}_{n}=\Delta+r_{n} \operatorname{det} A_{1}$

$\Delta=\left|\begin{array}{ll}A_{1} & \hat{a}_{2} \\ \hat{a}_{3} & l_{n}\end{array}\right|$.

But the elements of $\Delta$ satisfy (2.4), hence, by (2.5) and (2.7) we deduce that

$\Delta \geq \prod_{i=1}^{n}\left(a_{i i}-\hat{r}_{i}\right) \geq \prod_{i=1}^{n}\left(a_{i i}-r_{i}\right)=\prod_{i=1}^{n} l_{i}$,

And;

$\Delta \leq l_{n} \prod_{i=1}^{n-1}\left(a_{i i}+\hat{r}_{i}\right) \leq l_{n} \prod_{i=1}^{n-1}\left(l_{i}+2 r_{i}\right)$.

Also, by inductive assumption, since $A_{1}$, is of order $n-1$, and, by (2.5),

$r_{i} \geq \sum_{j=i+1}^{n}\left|a_{i j}\right| \geq \sum_{j=i+1}^{n-1}\left|a_{i j}\right|$

We have , using (2.10), (2.9) and (2.8),

$\mathbb{D}_{n} \geq \prod_{i=1}^{n} l_{i}+r_{n} \sum_{k=0}^{n-1}\left(\prod_{i=1}^{k} l_{i} \prod_{i=k+1}^{n-1} r_{i}\right)=\sum_{k=0}^{n}\left(\prod_{i=1}^{k} l_{i} \prod_{i=k+1}^{n-1} r_{i}\right)$,

and

$\mathbb{D}_{n} \leq l_{n} \prod_{i=1}^{n-1}\left(l_{i}+2 r_{i}\right)+r_{n} \sum_{k=0}^{n-1}\left(\prod_{i=1}^{k-1}\left(l_{i}+2 r_{i}\right) l_{k} \prod_{k+1}^{n-1} r_{i}\right.$

$=\sum_{k=0}^{n}\left(\prod_{i=1}^{k-1}\left(l_{i}+2 r_{i}\right) l_{k} \prod_{i=k+1}^{n} r_{i}\right.$.

\section{Remark 2.5}

Price (1951) [29], A. Ostrowski [30-32], Brenner [33, 34] and Schneider (1953) [35] have given lower and upper bounds for the absolute value of determinants satisfying more general condition than (2.4).

However, the above theorem is not implied by any of their results.

\section{- Bounds on norms of compound matrices}

Let $A$ be a matrix in $M_{n}(\mathbb{C})$, For subsets $\alpha$ and $\beta$ of $\{1, \ldots, n\}$ we denote by $A(\alpha \mid \beta)$ the sub-matrix of $A$ whose rows are indexed by $\alpha$ and whose columns are indexed by $\beta$ in their natural order.

Let $k$ be a positive integer, $k \leq n$. we denote by $C_{k}(A)$ the $k^{\text {th }}$ of the matrix $A$, that is, the $\left(\begin{array}{l}n \\ k\end{array}\right) \times\left(\begin{array}{l}n \\ k\end{array}\right)$ matrix whose elements are the minors det $A(\alpha \mid \beta) \alpha, \beta \subseteq\{1, \ldots, n\},|\alpha|=|\beta|=k$. We index $C_{k}(A)$ by $\alpha \subseteq\{1, \ldots, n\}$, $|\alpha|=k$ (ordered lexicographically).

- Example 2 if $A \in M_{3}(\mathbb{R})$ and $k=2$ then :

$$
C_{2}(A)=\left(\begin{array}{lll}
\operatorname{det} A(\{1,2\} \mid\{1,2\}) & \operatorname{det} A(\{1,2\} \mid\{1,3\}) & \operatorname{det} A(\{1,2\} \mid\{2,3\}) \\
\operatorname{det} A(\{1,3\} \mid\{1,2\}) & \operatorname{det} A(\{1,3\} \mid\{1,3\}) & \operatorname{det} A(\{1,3\} \mid\{2,3\}) \\
\operatorname{det} A(\{2,3\} \mid\{1,2\}) & \operatorname{det} A(\{2,3\} \mid\{1,3\}) & \operatorname{det} A(\{2,3\} \mid\{2,3\})
\end{array}\right)
$$

- Example 3 if $A \in M_{4}(\mathbb{R})$ and $k=3$ then: 


$$
C_{3}(A)=\left(\begin{array}{l}
\operatorname{det} A(1,2,3 \mid 1,2,3) \operatorname{det} A(1,2,3 \mid 1,2,4) \operatorname{det} A(1,2,3 \mid 1,3,4) \operatorname{det} A(1,2,3 \mid 2,3,4) \\
\operatorname{det} A(1,2,4 \mid 1,2,3) \operatorname{det} A(1,2,4 \mid 1,2,4) \operatorname{det} A(1,2,4 \mid 1,3,4) \operatorname{det} A(1,2,4 \mid 2,3,4) \\
\operatorname{det} A(1,3,4 \mid 1,2,3) \operatorname{det} A(1,3,4 \mid 1,2,4) \operatorname{det} A(1,3,4 \mid 1,3,4) \operatorname{det} A(1,3,4 \mid 2,3,4) \\
\operatorname{det} A(2,3,4 \mid 1,2,3) \operatorname{det} A(2,3,4 \mid 1,2,4) \operatorname{det} A(2,3,4 \mid 1,3,4) \operatorname{det} A(2,3,4 \mid 2,3,4)
\end{array}\right.
$$

The most important property of the compound mapping is that it is multiplicative.

Lemma 2.6. ([36], Theorem 19.F.2)

Let $A$ and $B$ be $n \times n$ matrices and let $1 \leq k \leq n$, then $C_{k}(A B)=C_{k}(A) C_{k}(B)$.

This property is equivalent to the Binet-Cauchy theorem :

Theorem 2.7 (Binet-Cauchy Theorem)

Let $A$ be a $n \times m$ complex matrix, $B$ be a $m \times l$ complex matrix and $p \leq \min \{n, m, l\}$ then $C_{p}(A B)=C_{p}(A) C_{p}(B)$.

Some other principal properties of compound matrices are given in [37- 40] for $A \in M_{n}(\mathbb{C})$ and $p$ an integer, $1 \leq p \leq n$ :

in particular, let $A \in M_{n}(\mathbb{C})$ and $k \leq n$ then we have :

$\bullet_{1}$ if $A$ is unitary, then $C_{k}(A)$ is unitary.

$\bullet_{2}$ if $A$ is diagonal, then $C_{k}(A)$ is diagonal.

$\bullet_{3}$ if $A$ is upper (lower) triangular, then $C_{k}(A)$ is upper (lower) triangular.

$\bullet_{4} C_{k}\left(A^{T}\right)=C_{k}(A)^{T}$.

$\bullet_{5} \operatorname{det}(A+I)=1+\operatorname{det}(A)+\sum_{i=1}^{n-1} \operatorname{tr}\left(C_{i}(A)\right.$.

${ }_{6}$ if $\left\{\lambda_{i}, i=1 \ldots \ldots, n\right\}$ are eigenvalues of $A$ then the eigenvalues of $A^{[k]}$ are of the following form :

$\left\{\lambda_{i_{1}}+\ldots+\lambda_{i_{k}}, 1 \leq i_{1}<\ldots<i_{k} . \leq n\right\}$.

${ }_{7}$ if $\left\{\lambda_{i}, i=1 \ldots \ldots, n\right\}$ are eigenvalues of $A$ then the eigenvalues of $C_{k}(A)$ are of the following form :

$\left\{\lambda_{i_{1}} \ldots \lambda_{i_{k}}, 1 \leq i_{1}<\ldots .<i_{k} . \leq n\right\}$.

The main use of compound matrices are their spectral properties which follow from the previous lemma together with the Jordan Canonical Form.

The compounds of companion matrices can be used to study products of roots of polynomials.

Now, let $v$ be a vector norm on $\mathbb{C}^{n}$, and for a positive integer $k, k \leq n$, let $\mu$ be a norm on $M_{m}(\mathbb{C})$ where $m=\left(\begin{array}{l}n \\ k\end{array}\right)$ then we have:

Theorem $2.7_{\text {bis }}$ (Elsner (1998) [41] Theorem 2.1)

$\mu\left(C_{k}(A)\right) \leq \theta_{k}(\mu, v) \max _{\alpha \subseteq\{1, \ldots, n\} ;|\alpha|=k} \prod_{i \in \alpha} v\left(\operatorname{col}_{i}(A)\right)$.

Where:

$\theta_{k}(\mu, v)=\max \left\{\mu\left(C_{k}(B)\right) ; B \in M_{n}\left(\mathbb{C}, v\left(\operatorname{col}_{i}(B)\right)=1, i=1, \ldots,,\right\}\right.$

with $\operatorname{col}_{i}(B)$ denotes the $i^{\text {th }}$ column of $B$.

- Some criteria of stability on matrices given by $\mathrm{Li}$ and Wang using the compound matrix and Lozinskii measure

Lemma 2.8 (Li-Wang [2])

(i) Let $\mu$ be a Lozinskĭ measure. Then $s(\mathbb{A}) \leq \mu(\mathbb{A})$

(ii) $s(A)=\inf \left\{\mu(A) ; \mu\right.$ is a Lozinskĭ measure on $\left.\mathbb{M}_{n}(\mathbb{K})\right\}$ where $\mathbb{K}=\mathbb{R}$ or $\mathbb{C}$. 


\section{Proof}

See Coppel (1965) [23] for (i) and Li-Wang [2], for (ii)

Proposition 2.9 (see Li-Wang [2]).

$s(\mathbb{A})<0 \Leftarrow s\left(\mathbb{A}^{[2]}\right)<0$ and $(-1)^{n} \operatorname{det}(\mathbb{A})>0$.

Theorem 2.10 (see Li-Wang [2])

Assume that $\mathbb{A} \in \mathbb{M}_{n}(\mathbb{R})$ and $(-1)^{n} \operatorname{det}(\mathbb{A})>0$. Then $\mathbb{A}$ is stable if and only if $\mu\left(A^{[2]}\right)<0$ for some Lozinskii measure $\mu$ on $\frac{M_{\frac{n(n-1)}{2}}}{2}(\mathbb{R})$

\section{Corollary 2.11}

Assume that $\mathbb{A} \in \mathbb{M}_{n}(\mathbb{R})$ and $(-1)^{n} \operatorname{det}(\mathbb{A})>0$. Then $\mathbb{A}$ is stable if the following conditions are verified:

- $\hat{a}_{j, j}+\sum_{i=1 ; i \neq j}^{\frac{n(n-1)}{2}}\left|\hat{a}_{i, j}\right|<0, \forall \quad j=1, \ldots \frac{n(n-1)}{2}$

where $\left(\hat{a}_{i, j}\right)_{i, j=1, \ldots} \frac{n(n-1)}{2}$ are the entries of seconde additive compound matrix $A^{[2]}$.

\section{Proof}

If we take as Lozinski ̌’'s measure $\mu\left(A^{[2]}\right)=\sup _{j}\left(\hat{a}_{j, j}+\sum_{i=1 ; i \neq j}^{\frac{n(n-1)}{2}}\left|\hat{a}_{i, j}\right|\right), \forall j=1, \ldots \frac{n(n-1)}{2}$

then by applying the above theorem $\mu\left(A^{[2]}\right)<0$ and $\mathbb{A}$ is stable.

\section{Definition 2.12}

A matrix $A=\left(a_{i j}\right) ; 1 \leq i, j \leq n$ is said to have dominant principal diagonal if

$\left|a_{i i}\right|>\sum_{k \neq i}^{n}\left|a_{i k}\right|$ for each $1 \leq i \leq n$.

\section{Lemma 2.13}

Let $A$ be a square real or complex matrix such that :

$\left|a_{i i}\right|>\sum_{k \neq i}^{n}\left|a_{i k}\right|$ for each $1 \leq i \leq n$.

Then $A$ is invertible and the set of its eigenvalues is included in $\bigcup_{i=1}^{n}\left\{z \in \mathbb{C} ;\left|z-a_{i i}\right| \leq \sum_{k \neq i}^{n}\left|a_{i k}\right|\right\}$.

\section{Proof}

Suppose that $A x=0$ admit a solution $x \neq 0$ where $x=\left(x_{1}, x_{2}, \ldots, x_{n}\right)^{T}$. Let $i_{0}$ such that $\left|x_{i_{0}}\right|=\max _{1 \leq i \leq n}\left|x_{i}\right|$.

The $i_{0}^{\text {th }}$ equation of te system $A x=0$ can be written as follow :

$\sum_{k=1}^{n} a_{i_{0} k} x_{k}=0$ or $a_{i_{0} i_{0}} x_{i_{0}}=\sum_{k \neq i_{0}}^{n}-a_{i_{0} k} x_{k}$

But $x_{i_{0}} \neq 0$ then $a_{i_{0} i_{0}}=\sum_{k \neq i_{0}}^{n}-a_{i_{0} k} \frac{x_{k}}{x_{i_{0}}}$ and $\left|a_{i_{0} i_{0}}\right|=\sum_{k \neq i_{0}}^{n}\left|a_{i_{0} k}\right| \frac{\left|x_{k}\right|}{\mid x_{i_{0}}}\left|\leq \sum_{k \neq i_{0}}^{n}\right| a_{i_{0} k} \mid$ which is impossible.

Now, let $z$ is an eigenvalue of $A$ then $A-z I$ is not invertible.

It follows that it is not dominant principal diagonal in particular there exists $i$ such that $\left|a_{i i}-z\right| \leq \sum_{k=1, k \neq i}^{n}\left|a_{i k}\right|$ and $z \in \bigcup_{i=1}^{n}\left\{z \in \mathbb{C} ;\left|z-a_{i i}\right| \leq \sum_{k \neq i}^{n}\left|a_{i k}\right|\right\}$

\section{Remark 2.14}

(i) If $A$ is a matrix with dominant principal diagonal, then $\rho\left(I-D^{-1} A\right)<1$ where $D$ is the diagonal of $A$ and $\rho\left(I-D^{-1} A\right)$ is the spectral radius of $I-D^{-1} A$ which is defined as the maximum of the moduli $|\lambda|$ of eigenvalues $\lambda$ of $I-D^{-1} A$.

(ii) $\mu\left(A^{[2]}\right)<0$ can be interpreted as $\hat{a}_{j, j}<0$ for $j=1, \ldots, \frac{n(n-1)}{2}$, and $A^{[2]}$ is diagonally dominant in columns.

\section{- Positive Definite Matrix}

\section{Definition 2.15}

An $n \times n$ complex matrix $A$ is called positive definite if 
for all nonzero complex vectors $x \in \mathbb{C}^{n}$, where $x^{*}$ denotes the conjugate transpose of the vector $x$.

In the case of a real matrix $A$, equation (2.4) reduces to

$\Re e\left[x^{T} A x\right]>0$

Where $x^{T}$ denotes the transpose.

- Positive definite matrices are of both theoretical and computational importance in a wide variety of applications. They are used, for example, in optimization algorithms and in the construction of various linear regression models (Johnson 1970) [42].

A positive definite matrix has at least one matrix square root. Furthermore, exactly one of its matrix square roots is itself positive definite.

A necessary and sufficient condition for a complex matrix $A$ to be positive definite is that the Hermitian part

$A_{H}=\frac{1}{2}\left(A+A^{H}\right)$

where $A^{H}$ denotes the conjugate transpose, be positive definite.

This means that a real matrix $A$ is positive definite iff the symmetric part

$A_{S}=\frac{1}{2}\left(A+A^{T}\right)$

where $A^{T}$ is the transpose, is positive definite (Johnson 1970 [42]).

- Confusingly, the discussion of positive definite matrices is often restricted to only Hermitian matrices, or symmetric matrices .

In the case of real matrices (Pease (1965) [43]; Johnson (1970) [42]; Marcus and Minc (1988) [44]; Marcus and Minc (1992) [45]; Golub and Van Loan (1996) [46]).

A Hermitian (or symmetric) matrix is positive definite iff all its eigenvalues are positive. Therefore, a general complex (respectively, real) matrix is positive definite iff its Hermitian (or symmetric) part has all positive eigenvalues.

The determinant of a positive definite matrix is always positive, so a positive definite matrix is always nonsingular.

\section{Definition 2.16}

(1) An real square matrix $A$ is said Z-matrix if their of diagonal elements are all non-positive.

(1) An real square matrix $A$ is said $M$-matrix if it is $Z$-matrix and fulfilling one of the conditions of the following theorem of Fiedler and Ptàk [47].

Theorem 2.17 (Fiedler-Ptàk)

Let $A$ be a Z-matrix. Then the following conditions are equivalent to each other :

$1^{o}$ There exists a vector $x \geq 0$ such that $A x>0$;

$2^{\circ}$ there exists a vector $x>0$ such that $A x>0$;

$3^{o}$ there exists a diagonal matrix $D$ with positive diagonal elements such that $A D e>0$ (here $e$ is the vector whose all coordinates are 1);

$4^{o}$ there exists a diagonal matrix $D$ with positive diagonal elements such that the matrix $W=A D$ is a matrix with dominant positive principal diagonal;

$5^{o}$ for each diagonal matrix $R$ such that $R \geq A$ the inverse $R^{-1}$ exists and $\rho\left(R^{-1}(P-A)\right)<1$, where $P$ is the diagonal of $A$;

$6^{\circ}$ if $B$ is a Z-matrix and $B \geq A$, then $B^{-1}$ exists;

$7^{\circ}$ each eigenvalue of $A$ is positive;

$8^{o}$ all principal minors of $A$ are positive;

$9^{o}$ there exists a strictly increasing sequence $0 \neq M_{1} \subset M_{2} \subset \ldots \ldots M_{n}$ such that the principal minors $\operatorname{det} A\left(M_{i}\right)$ are positive;

$10^{\circ}$ there exists a permutation matrix $\mathcal{P}$ such that $\mathcal{P} A \mathcal{P}^{-1}$ may be written in the form $R S$ where $R$ is a lower 
triangular matrix positive diagonal elements such that $R$ is a Z-matrix and $S$ is an upper triangular matrix with positive diagonal elements such that $S$ is a Z-matrix;

$11^{o}$ the inverse $A^{-1}$ exists and $A^{-1} \geq 0$;

$12^{\circ}$ the real part of each eigenvalue of $A$ is positive;

$13^{o}$ for each vector $x \neq 0$ there exists an index $k$ that $x_{k} y_{k}>0$ for $y=A x$

Proof : See Fiedler-Ptàk (1962) [47].

Theorem 2.18 (A caracterization of M-matrices in relation to the definite positive matrices)

Let $A$ be a square matrix of order $n$ satisfying: $a_{i i} \geq 0 ; a_{i j} \leq 0$ if $i \neq j$. Then the following conditions are equivalent:

(i) $A^{-1}$ exists and its elements are $\geq 0$.

(ii) There exists a diagonal matrix $D$ with elements $>0$ such that $D A$ is definite positive matrix.

\section{Proof}

(i) $\Rightarrow(i i)$

Let $e$ be the vector having all its components equal to 1 . We define $x$ and $y$ by $A x=e$ and $A^{*} y=e$. Then we have $x_{i}>0$ and $y_{i}>0$ for all $i$.

Let $B$ be the matrix defined by $b_{i j}=y_{i} a_{i j} x_{j}$. Then $b_{i i}-\sum_{j \neq i}\left|b_{i j}\right|=\sum_{j} b_{i j j}=y_{i}>0$ and $b_{i i}-\sum_{j \neq i}\left|b_{j i}\right|=$ $\sum_{j} b_{j i j}=x_{i}>0$.

Consequently $B$ and $B^{*}$ are strictly dominant diagonal matrices. Then $B+B^{*}$ is also a strictly dominant diagonal matrix and it is a definite positive matrix because it is symmetric. Then there exists $\alpha>0$ such that $\langle B u, u>\geq$ $\alpha\|u\|^{2}$.

Now, let $d_{i}=\frac{y_{i}}{x_{i}}$ and $d_{i}$ are the elements the diagonal matrix $D$ then we have

$<D A u, u>=\sum_{i j} d_{i} a_{i j} u_{i} u_{j}=\sum_{i j} b_{i j} \frac{u_{i}}{x_{i}} \frac{u_{j}}{x_{j}} \geq \alpha \sum_{i}\left|\frac{u_{i}}{x_{i}}\right|^{2} \beta \sum_{i}\left|u_{i}\right|^{2}$.

(ii) $\Rightarrow(i)$

If $u \neq 0$ and $A u=v$ then we have $\sum_{i} d_{i} u_{i} v_{i}>0$.

Consequently, there exists $i$ such that $u_{i} v_{i}>0$ which entails $(i)$ by applying the property $13^{o}$ of above theorem on the M-matrices.

- The Schur stability criteria of matrices using the additive compound matrix

Definition 2.19 (Shur stability)

A matrix $A$ is said to be Schur stable if $\rho(A)<1$, where $\rho(A)=\max \{|\lambda| ; \lambda \in \sigma(A)\}$ (the spectral radius of $A$ ).

Consider the $\mathcal{C}^{r} ; r \geq 1$ map:

$x \rightarrow g(x) ; x \in \mathbb{R}^{n}$

If (2.8) has a fixed point $x=x^{*}$, that is, $x^{*}=g\left(x^{*}\right)$, then the linear map corresponding to $((\mathbf{2 . 8})$ is

$y \rightarrow A y ; y \in \mathbb{R}^{n}$

Where $A=D g\left(x^{*}\right)$, the Jacobian matrix of $g$ at $x^{*}$.

Lemma 2.20 (see Liao (2002) [48] Theorem 2.1).

If the matrix $A$ of the system (2.9) is Schur stable, then the fixed point $x^{*}$ of the system (2.9) is asymptotically stable. that is the eigenvalues of $A$ have strictly negative real part.

Theorem 2.21 (see Zhang and Zheng (2013) [49])

$\star_{1}$ Let $\left.B=I+\frac{2}{\operatorname{det}(A-I)}(A-I)^{-1}\right)$, if $(-1)^{n} \operatorname{det}(B)>0$, then $A$ is Schur stable $\Leftarrow$ there exists some Lozinskii measure $\mu$ such that $\mu\left(B^{[2]}\right)<0$.

$\star_{2}$ Let $A \in M_{n}(\mathbb{R})$, then $\rho(A)<1 \Leftarrow \rho\left(C_{2}(A)\right)<1$ and $\operatorname{det}\left(I-A^{2}\right)>0$.

$\star_{3}$ Let $A \in M_{n}(\mathbb{R})$, then $\rho(A)<1 \Leftarrow \sigma_{1} \sigma_{2}<1$ and $\operatorname{det}\left(I-A^{2}\right)>0$. 
Where $\left\{\sigma_{1}, \sigma_{2}, \ldots, \sigma_{n}\right\}$ are the singular values of $A$, i.e the eigenvalues of the symmetric matrix $\sqrt{A^{*} A}$ such that $\sigma_{1} \geq \sigma_{2} \geq \ldots \ldots \geq \sigma_{n} \geq 0$.

In next section we give some preliminary definitions and lemmas for linear stability of above system.

\section{Some Preliminary Definitions and Lemmas}

- Writting the above five-dimensional system as follow:

$x^{\prime}(t)=f(x(t))$ where $x(t)=\left(x_{1}, x_{2}(t), x_{3}, x_{4}, x_{5}\right)^{T}=(E(t), I(t), C(t), H(t), D(t))^{T}$ and

$f=\left(f_{1}, f_{2}, f_{3}, f_{4}, f_{5}\right)^{T}$ such that:

$f_{1}: \mathbb{R}^{5} \rightarrow \mathbb{R} ; f_{1}[x(t)]=B-\mu E(t)+\beta_{9} H(t)+\left(\beta_{10}-\beta_{1}\right) E(t) I(t)+\beta_{7} E(t) D(t)$

$f_{2}: \mathbb{R}^{5} \rightarrow \mathbb{R} ; f_{2}[x(t)]=-\left(\beta_{2}+\beta_{6}+\beta_{8}+\mu\right) I(t)+\left(\beta_{1}-\beta_{10}\right) E(t) I(t)$

$f_{3}: \mathbb{R}^{5} \rightarrow \mathbb{R} ; f_{3}[x(t)]=\beta_{2} I(t)-\left(\beta_{5}+\beta_{3}+\mu\right) C(t)+\beta_{4} H(t)$

$f_{4}: \mathbb{R}^{5} \rightarrow \mathbb{R} ; f_{4}[x(t)]=\beta_{8} I(t)+\beta_{3} C(t)-\left(\beta_{4}+\beta_{9}+\mu\right) H$

$f_{5}: \mathbb{R}^{5} \rightarrow \mathbb{R} ; f_{5}[x(t)]=\beta_{6} I(t)+\beta_{5} C(t)-\beta_{7} D(t) E(t)$

where the $\left\langle<{ }^{T}\right\rangle>$ denotes transpose.

\section{- Basic reproduction number}

Mathematical modeling can play an important role in helping to quantify possible disease control strategies by focusing on the important aspects of a disease, determining threshold quantities for disease survival, and evaluating the effect of particular control strategies.

A very important threshold quantity is the basic reproduction number, sometimes called the basic reproductive number or basic reproductive ratio (Heffernan et al. 2005 [6]), which is usually denoted by $\mathcal{R}_{0}$.

The epidemiological definition of $\mathcal{R}_{0}$ is the average number of secondary cases produced by one infected individual introduced into a population of susceptible individuals, where an infected individual has acquired the disease, and susceptible individuals are healthy but can acquire the disease.

In reality, the value of $\mathcal{R}_{0}$ for a specific disease depends on many variables, such as location and density of population.

The study of the stability of jacobian matrices of order less than three of a dynamic system yields a reasonable $\mathcal{R}_{0}$, but for more complex compartmental models, especially those with more infected compartments, the study of the stability is difficult as it relies on the algebraic Routh-Hurwitz conditions for stability of the Jacobian matrix.

An alternative method proposed by Diekmann et al. (1990) [7] and elaborated by van den Driessche and Watmough (2002) [8] gives a way of determining $\mathcal{R}_{0}$ for a compartmental model by using the next generation matrix.

Here an outline of this method is given, the proofs and further details can be found in van den Driessche and Watmough (2002) and van den Driessche and Watmough (2008) [50].

Let $x=\left(x_{1}, x_{2}, \ldots, x_{m}, \ldots, x_{n}\right)^{T}$ be the number of individuals in each compartment, where the first $m<n$ compartments contain infected individuals.

Assume that the equilibrium point $x^{*}$ exists and is stable in the absence of disease, and that the linearized equations for $x_{1}, \ldots, x_{m}$ at the $x^{*}$ decouple from the other equations. The assumptions are given in more details in the references cited above.

Consider these equations written in the form:

$\frac{d x_{i}}{d t}=\mathfrak{F}_{i}(x)-\mathcal{V}_{i}(x), 1 \leq i \leq m$

In this splitting,

$\mathfrak{F}_{i}(x)$ is the rate of appearance of new infections in compartment $i$,

and

$\mathcal{V}_{i}(x)$ is the rate of other transitions between compartment $i$ and other infected compartments.

It is assumed that $\mathfrak{F}_{i}, \mathcal{V}_{i} \in \mathcal{C}^{2}$ and $\mathfrak{F}_{i}=0, m+1 \leq i \leq n$

\section{Remark 3.1}

Let $n=5$ and $\left(x_{1}, x_{2}, \ldots, x_{5}\right)^{T}=(E, I, C, H, D)^{T}$ the compments of our system Covid-19 then we have : 
- $m=2$

- $\left\{\begin{array}{l}\mathfrak{F}_{1}(E, I, C, H, D)=\beta_{7} E D+\beta_{10} E I \\ \mathfrak{F}_{2}(E, I, C, H, D)=\beta_{1} E I \\ \mathfrak{F}_{i}(E, I, C, H, D)=0 ; 3 \leq i \leq 5\end{array}\right.$

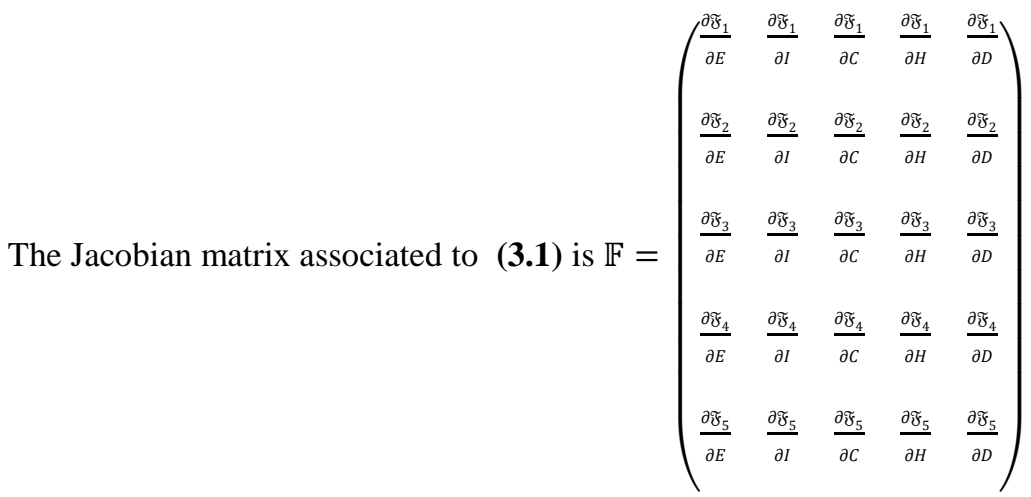

$=\left(\begin{array}{lllll}\beta_{7} D+\beta_{10} I & \beta_{10} E & 0 & 0 & \beta_{7} E \\ \beta_{1} I & \beta_{1} E & 0 & 0 & 0 \\ 0 & 0 & 0 & 0 & 0 \\ 0 & 0 & 0 & 0 & 0 \\ 0 & 0 & 0 & 0 & 0\end{array}\right)$

And for $m=2$ we have:

- $\mathbb{F}_{m}=\left(\begin{array}{ll}\beta_{7} D+\beta_{10} I & \beta_{10} E \\ \beta_{1} I & \beta_{1} E\end{array}\right)$

- $\left\{\begin{array}{l}\mathcal{V}_{1}(E, I, C, H, D)=-B+\beta_{1} E I-\beta_{9} H+\mu E \\ \mathcal{V}_{2}(E, I, C, H, D)=\left(\beta_{2}+\beta_{6}+\beta_{8}+\mu\right) I+\beta_{10} E I \\ \mathcal{V}_{3}(E, I, C, H, D)=-\beta_{2} I+\left(\beta_{5}+\beta_{3}+\mu\right) C-\beta_{4} H \\ \mathcal{V}_{4}(E, I, C, H, D)=-\beta_{8} I-\beta_{3} C+\left(\beta_{4}+\beta_{9}+\mu\right) H \\ \mathcal{V}_{5}(E, I, C, H, D)=\beta_{6} I-\beta_{5} C+\beta_{7} D E\end{array}\right.$

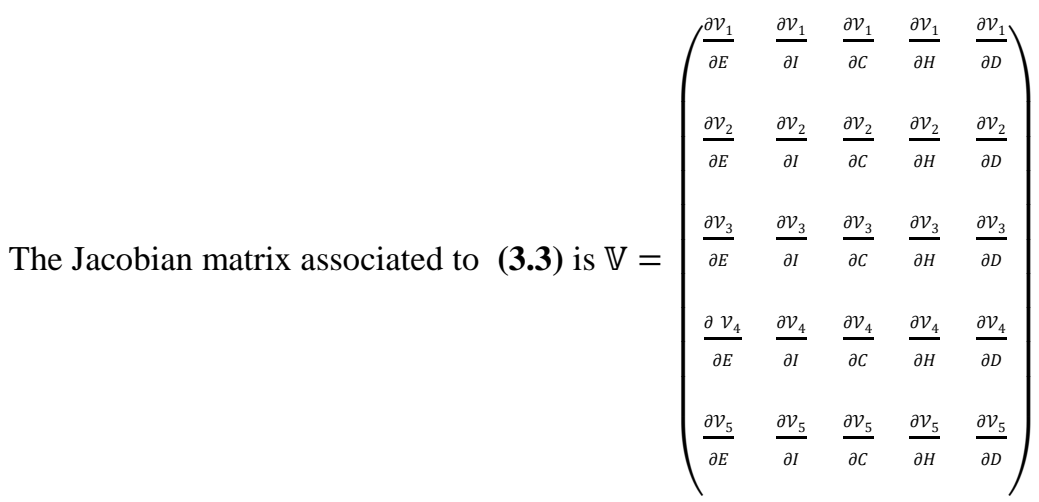




$$
=\left(\begin{array}{lllll}
\beta_{1} I+\mu & \beta_{1} E & 0 & -\beta_{9} & 0 \\
\beta_{10} I & \beta_{2}+\beta_{6}+\beta_{8}+\mu+\beta_{10} E & 0 & 0 & 0 \\
0 & -\beta_{2} & \beta_{5}+\beta_{3}+\mu & -\beta_{4} & 0 \\
0 & -\beta_{8} & -\beta_{3} & \beta_{4}+\beta_{9}+\mu & 0 \\
\beta_{7} D & -\beta_{6} & -\beta_{5} & 0 & \beta_{7} E
\end{array}\right)
$$

and for $m=2$ we have

- $\mathcal{V}_{m}=\left(\begin{array}{ll}\beta_{1} I+\mu & \beta_{1} E \\ \beta_{10} I & \beta_{2}+\beta_{6}+\beta_{8}+\mu+\beta_{10} E\end{array}\right)$

\section{- Important case}

Let $E=\frac{B}{\mu}, I=C=H=D=0$ and $\alpha=\beta_{2}+\beta_{6}+\beta_{8}+\mu$ then we have :

- $\mathbb{F}_{m}=\left(\begin{array}{cc}0 & \frac{\beta_{10} B}{\mu} \\ 0 & \frac{\beta_{1} B}{\mu}\end{array}\right), \bullet \mathcal{V}_{m}=\left(\begin{array}{cc}\mu & \beta_{1} \frac{B}{\mu} \\ 0 & \alpha+\frac{\beta_{10} B}{\mu}\end{array}\right), \bullet \mathcal{V}_{m}^{-1}=\left(\begin{array}{cc}\frac{1}{\mu} & \frac{-\beta_{1} B}{\mu\left[\beta_{10} B+\alpha \mu\right]} \\ 0 & \frac{1}{\alpha+\frac{\beta_{10} B}{\mu}}\end{array}\right)$ and $\bullet \mathbb{F}_{m} \mathcal{V}_{m}^{-1}=\left(\begin{array}{cc}0 & \frac{\beta_{10} B}{\alpha \mu+\beta_{10} B} \\ 0 & \frac{\beta_{1} B}{\alpha \mu+\beta_{10} B}\end{array}\right)$

The eigenvalues of $\mathbb{F}_{m} \mathcal{V}_{m}^{-1}$ are $\lambda_{0}=0$ and $\bullet \mathcal{R}_{0}=\frac{\beta_{1} B}{\alpha \mu+\beta_{10} B}=\rho\left(\mathbb{F}_{m} \mathcal{V}_{m}^{-1}\right)$ which is called effective basic reproduction number.

These following figures give the curves of $\mathcal{R}_{0}$-evolution with respect $\mu$ as abscissa of step $\Delta \mu=0.015$ and parameter $\beta_{10}$ but the other parameters are fixed as in above table.

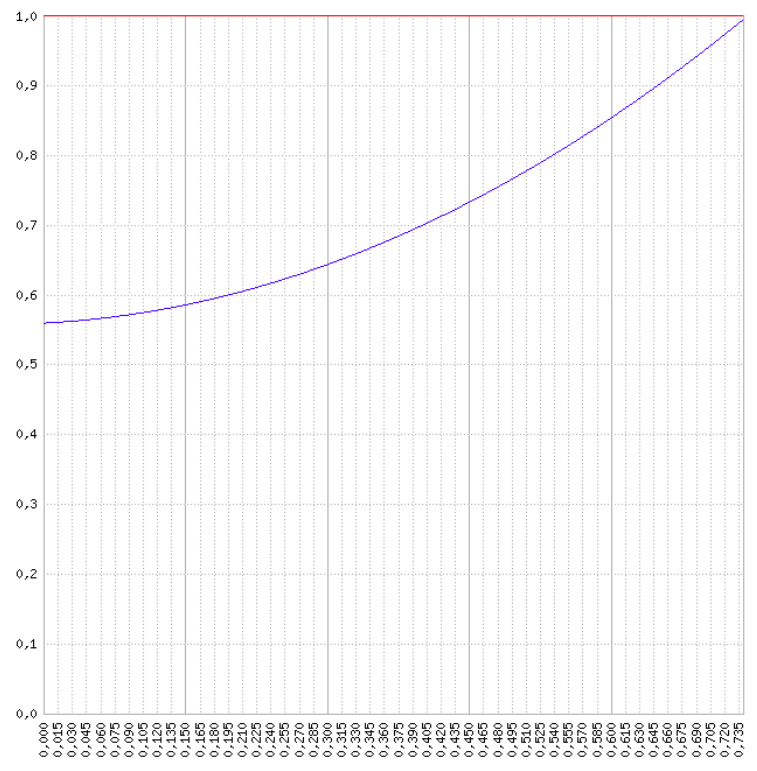

(a) $\mu \in[0,0.74] ; \beta_{10}=0.1$

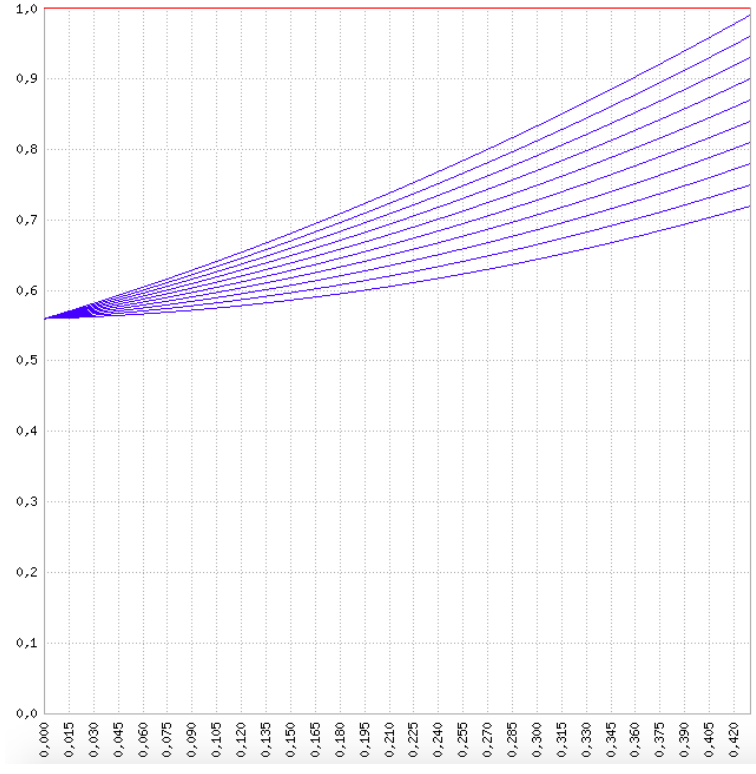

(b) $\mu \in[0,043] ; \beta_{10} \in[0.1,1] ; \Delta \beta_{10}=0.1$ 


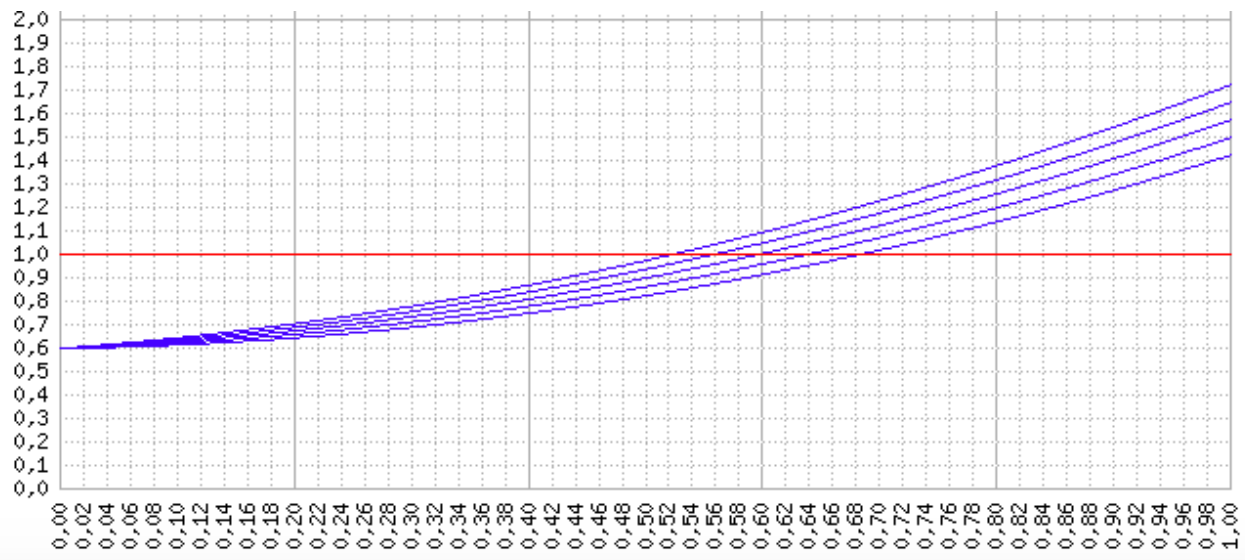

(c) $\beta_{10} \in[0.1,1] ; \Delta \beta_{10}=0.1$

\section{Remark 3.2}

Let $A=\left(a_{i j}\right)$ be a $n \times n$ real matrix such that $\left(a_{i j}\right) \leq 0$ for all $i \neq j, 1 \leq i, j \leq n$. Then matrix $A$ is also an Mmatrix if it can be expressed in the form $A=s I-B$, where $B=\left(b_{i j}\right)$ with $b_{i j} \geq 0$, for all $1 \leq i, j \leq n$, where $s$ is at least as large as the maximum of the moduli of the eigenvalues of $B$, and $I$ is an identity matrix.

For the non-singularity of $A$, according to the Perron-Frobenius theorem, it must be the case that $s>\rho(B)$. Also, for a non-singular M-matrix, the diagonal elements $a_{i i}$ of $A$ must be positive. Here we will further characterize only the class of non-singular M-matrices.

\section{Definition 3.3 (Metzler matrix)}

In mathematics, especially linear algebra, a matrix is called Metzler, quasipositive (or quasi-positive) or essentially nonnegative if all of its elements are non-negative except for those on the main diagonal, which are unconstrained. That is, a Metzler matrix is any matrix A which satisfies $A=\left(a_{i j}\right) ; \quad a_{i j} \geq 0, \quad i \neq j$.

$M$-matrices are very useful. We can found some of their applications to ecology, numerical analysis, probability, mathematical programming, game theory, control theory, and matrix theory.

\section{- Some fondamental properties of M-matrices}

An M-matrix $A \in M_{n}(\mathbb{R})$ is a matrix of the form $A=\alpha I-B$, where $B \geq 0$ ( $B$ is elementwise nonnegative) and $\alpha \geq \rho(B)$. (By the Perron-Frobenius theorem e.g., Intissar (2019) [51], $\rho(B)$, the spectral radius of $B$, is an eigenvalue of $B$.)

If $A=\alpha I-B$, where $I$ is the identity matrix, $B$ is non-negative and $\alpha>\rho(B)$, then $A$ is a non-singular M-matrix; if $\alpha=\rho(B)$, then $A$ is a singular M-matrix.

There are many definitions of M-matrices equivalent to the above. For example, if a matrix $A$ has the $Z$ sign pattern and $\rho(A)>0$, then $A$ is a non-singular M-matrix [5].

A matrix of the form $\alpha I-B, B \geq 0$ is called a $Z-$ matrix.

- Observe that a $Z$-matrix $A$ is an $M$-matrix if and only if $A+\epsilon l$ is nonsingular for all $\epsilon>0$.

We said that a matrix $A=\left(a_{i j}\right)$ of order $n$ has the $Z$ sign pattern if $a_{i j} \leq 0$ for all $i \neq j$.

If a matrix $A$ has the $Z$ sign pattern and $\rho(A)>0$, then $A$ is a non-singular M-matrix [52].

From Exercise $6 b$ of Horn and Johnson (1991) [53] and Berman and Plemmons (1979) [52], we get the following lemma :

\section{Lemma 3.4}

Let $A$ be a non-singular M-matrix and suppose $B$ and $B A^{-1}$ have the $Z$ sign pattern.Then $B$ is a non-singular Mmatrix if and only if $B A^{-1}$ is a non-singular M-matrix.

In general, this lemma does not hold if $B$ a singular M-matrix. It can be shown to hold if $B$ is singular and irreducible. However, this is not sufficient for our needs in part II of this work. we shall need of the following lemma :

\section{Lemma 3.5}

Let $A$ be a non-singular M-matrix and suppose $B \geq 0$.Then,

(i) $(A-B)$ is a non-singular M-matrix if and only $A-B) A^{-1}$ is a non-singular M-matrix. 
(ii) $(A-B)$ is a non-singular M-matrix if and only $A-B) A^{-1}$ is a non-singular M-matrix.

Proof

Let $C=A-B$ ). Then both $C$ and $C A^{-1}=I-B A^{-1}$ have the $Z$ sign pattern. (Recall that $A^{-1} \geq 0$ since $A$ is a nonsingular M-matrix).

Hence, the above lemma implies statement (i). A separate continuity argument can be constructed for each implication in the singular case.

The following theorem collects conditions that characterize nonsingular $M$-matrices.

\section{Theorem 3.6}

Let $A=\alpha l-B, B \geq 0$. Then the following statements are equivalent:

a. $\alpha>\rho(B)$,

b. $A$ is positive stable: If $\lambda$ is an eigenvalue of $A$, then $\Re e \lambda>0$,

c. $A$ is nonsingular and $A^{-1} \geq 0$,

d. $A x$ is positive for some positive vector $x$,

e. The principal minors of $A$ are positive,

f. The leading principal minors of $A$ are positive.

Proof

Conditions (b), (c), and (e) are due to Ostrowski [30], who introduced the concept of $M$-matrices. Condition (e) is known in the economics literature as the Hawkins-Simon condition [54].

Condition (d) is due to Schneider (1953) [35] and Ky-Fan (1958) [55] and the condition (f) to Fiedler and Ptak (1962) [47].

Many additional characterizations of nonsingular (and of singular) M-matrices are given in Berman and Plemmons (1979) [52].

A subset of the set of all M-matrices that contains the nonsingular M-matrices and whose matrices share many of their properties is the set of group-invertible M-matrices (M-matrices with "property c").

Basic reproduction number $\mathcal{R}_{0}$ for the model can be established using the next generation matrix method [56] and [7].

\section{Definition 3.7}

The basic reproduction number $\mathcal{R}_{0}$ is obtained as the spectral radius of matrix $\mathbb{F V}^{-1}$ at disease free equilibrium point. Where $\mathbb{F}$ and $\mathbb{V}$ are constructed as below:

$$
\mathbb{F}=\left(\frac{\partial \widetilde{r}_{i}\left(x^{*}\right)}{\partial x_{j}}\right)_{i j} \text { and } \mathbb{V}=\left(\frac{\partial \mathcal{V}_{i}\left(x^{*}\right)}{\partial x_{j}}\right)_{i j} \text { for } 1 \leq i, j \leq m
$$

For our system the graph of $\mathcal{R}_{0}$ with respect $\frac{1}{\mu}$ is :

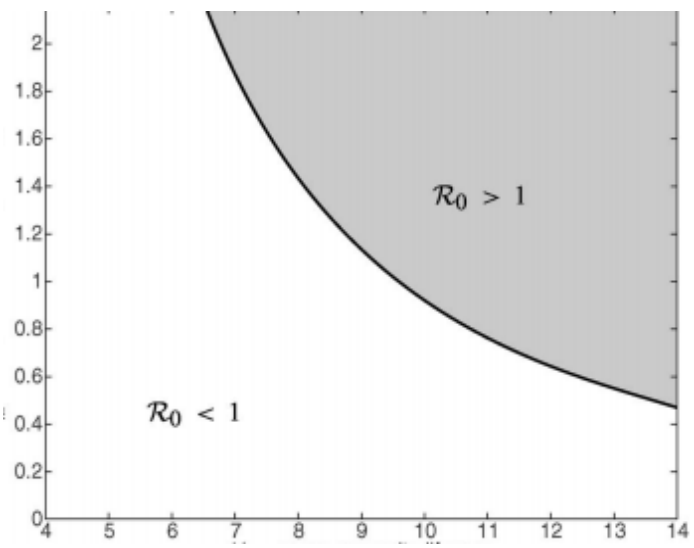

Basic reproduction number of infections $\mathcal{R}_{0}$ as a function of $\frac{1}{\mu}$. All other parameters are fixed. 
From the above functions $\left(f_{i}\right), 1 \leq i \leq 5$ of our system, we consider the associated functions $\left(\hat{f}_{i}\right), 1 \leq i \leq 5$ where we delete the linear elements and the negative nonlinear elements, i.e :

$$
\begin{aligned}
f_{1}[x(t)] \rightarrow \hat{f}_{1}[x(t)] & =\beta_{10} E(t) I(t)+\beta_{7} E(t) D(t) \\
f_{2}[x(t)] \rightarrow \hat{f}_{2}[x(t)] & =\beta_{1} E(t) I(t) \\
f_{3}[x(t)] \rightarrow \hat{f}_{3}[x(t)] & =0 \\
f_{4}[x(t)] \rightarrow \hat{f}_{4}[x(t)] & =0 \\
f_{5}[x(t)] \rightarrow \hat{f}_{5}[x(t)] & =0
\end{aligned}
$$

And the associated functions $\left(\hat{g}_{i}\right), 1 \leq i \leq 5$ where we delete the non negative nonlinear elements and we take the opposite of the obtained expression, i.e :

$$
\begin{aligned}
& f_{1}[x(t)] \rightarrow \hat{g}_{1}[x(t)]=-B+\mu E(t)-\beta_{9} H(t)+\beta_{1} E(t) I(t) \\
& f_{2}[x(t)] \rightarrow \hat{g}_{2}[x(t)]=+\left(\beta_{2}+\beta_{6}+\beta_{8}+\mu\right) I(t)+\beta_{10} E(t) I(t) \\
& f_{3}[x(t)] \rightarrow \hat{g}_{3}[x(t)]=-\beta_{2} I(t)+\left(\beta_{5}+\beta_{3}+\mu\right) C(t)-\beta_{4} H(t) \\
& f_{4}[x(t)] \rightarrow \hat{g}_{4}[x(t)]=-\beta_{8} I(t)-\beta_{3} C(t)+\left(\beta_{4}+\beta_{9}+\mu\right) H \\
& f_{5}[x(t)] \rightarrow \hat{g}_{5}[x(t)]=-\beta_{6} I(t)-\beta_{5} C(t)+\beta_{7} D(t) E(t)
\end{aligned}
$$

We define the matrices $\mathbb{F}$ and $\mathbb{V}$ as follow :

$$
\mathbb{F}=\left(\begin{array}{lllll}
\frac{\partial \hat{f}_{1}}{\partial x_{1}} & \frac{\partial \hat{f}_{1}}{\partial x_{2}} & \frac{\partial \hat{f}_{1}}{\partial x_{3}} & \frac{\partial \hat{f}_{1}}{\partial x_{4}} & \frac{\partial \hat{f}_{1}}{\partial x_{5}} \\
\frac{\partial \hat{f}_{2}}{\partial x_{1}} & \frac{\partial \hat{f}_{2}}{\partial x_{2}} & \frac{\partial \hat{f}_{2}}{\partial x_{3}} & \frac{\partial \hat{f}_{2}}{\partial x_{4}} & \frac{\partial \hat{f}_{2}}{\partial x_{5}} \\
\frac{\partial \hat{f}_{3}}{\partial x_{1}} & \frac{\partial \hat{f}_{3}}{\partial x_{2}} & \frac{\partial \hat{f}_{3}}{\partial x_{3}} & \frac{\partial \hat{f}_{3}}{\partial x_{4}} & \frac{\partial \hat{f}_{3}}{\partial x_{5}} \\
\frac{\partial \hat{f}_{4}}{\partial x_{1}} & \frac{\partial \hat{f}_{4}}{\partial x_{2}} & \frac{\partial \hat{f}_{4}}{\partial x_{3}} & \frac{\partial \hat{f}_{4}}{\partial x_{4}} & \frac{\partial \hat{f}_{4}}{\partial x_{5}} \\
\frac{\partial \hat{f}_{5}}{\partial x_{1}} & \frac{\partial \hat{f}_{5}}{\partial x_{2}} & \frac{\partial \hat{f}_{5}}{\partial x_{3}} & \frac{\partial \hat{f}_{5}}{\partial x_{4}} & \frac{\partial \hat{f}_{5}}{\partial x_{5}}
\end{array}\right) \text { and } \mathbb{V}=\left(\begin{array}{llllll}
\frac{\partial \hat{g}_{1}}{\partial x_{1}} & \frac{\partial \hat{g}_{1}}{\partial x_{2}} & \frac{\partial \hat{g}_{1}}{\partial x_{3}} & \frac{\partial \hat{g}_{1}}{\partial x_{4}} & \frac{\partial \hat{g}_{1}}{\partial x_{5}} \\
\frac{\partial \hat{g}_{2}}{\partial x_{1}} & \frac{\partial \hat{g}_{2}}{\partial x_{2}} & \frac{\partial \hat{g}_{2}}{\partial x_{3}} & \frac{\partial \hat{g}_{2}}{\partial x_{4}} & \frac{\partial \hat{g}_{2}}{\partial x_{5}} \\
\frac{\partial \hat{g}_{3}}{\partial x_{1}} & \frac{\partial \hat{g}_{3}}{\partial x_{2}} & \frac{\partial \hat{g}_{3}}{\partial x_{3}} & \frac{\partial \hat{g}_{3}}{\partial x_{4}} & \frac{\partial \hat{g}_{3}}{\partial x_{5}} \\
\frac{\partial \hat{g}_{4}}{\partial x_{1}} & \frac{\partial \hat{g}_{4}}{\partial x_{2}} & \frac{\partial \hat{g}_{4}}{\partial x_{3}} & \frac{\partial \hat{g}_{4}}{\partial x_{4}} & \frac{\partial \hat{g}_{4}}{\partial x_{5}} \\
\frac{\partial \hat{g}_{5}}{\partial x_{1}} & \frac{\partial \hat{g}_{5}}{\partial x_{2}} & \frac{\partial \hat{g}_{5}}{\partial x_{3}} & \frac{\partial \hat{g}_{5}}{\partial x_{4}} & \frac{\partial \hat{g}_{5}}{\partial x_{5}} \\
& & & & &
\end{array}\right)
$$

\section{Remark 3.8}

(i) The explicit matrix $\mathbb{F}$ is :

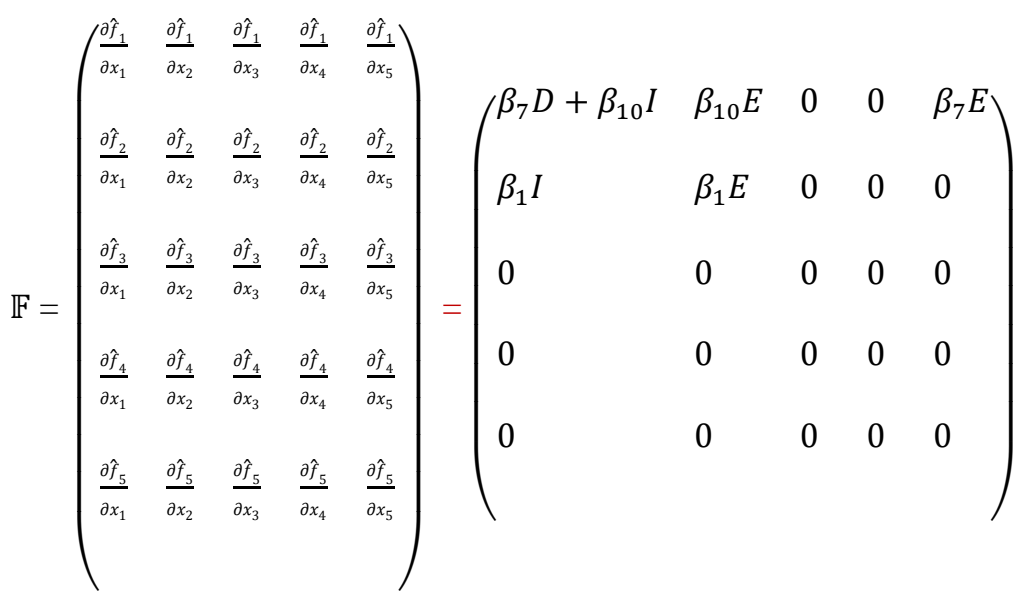

(ii) The explicit matrix $\mathbb{V}$ is : 


$$
\begin{aligned}
& \mathbb{V}=\left(\begin{array}{lllll}
\frac{\partial \hat{g}_{1}}{\partial x_{1}} & \frac{\partial \hat{g}_{1}}{\partial x_{2}} & \frac{\partial \hat{g}_{1}}{\partial x_{3}} & \frac{\partial \hat{g}_{1}}{\partial x_{4}} & \frac{\partial \hat{g}_{1}}{\partial x_{5}} \\
\frac{\partial \hat{g}_{2}}{\partial x_{1}} & \frac{\partial \hat{g}_{2}}{\partial x_{2}} & \frac{\partial \hat{g}_{2}}{\partial x_{3}} & \frac{\partial \hat{g}_{2}}{\partial x_{4}} & \frac{\partial \hat{g}_{2}}{\partial x_{5}} \\
\frac{\partial \hat{g}_{3}}{\partial x_{1}} & \frac{\partial \hat{g}_{3}}{\partial x_{2}} & \frac{\partial \hat{g}_{3}}{\partial x_{3}} & \frac{\partial \hat{g}_{3}}{\partial x_{4}} & \frac{\partial \hat{g}_{3}}{\partial x_{5}} \\
\frac{\partial \hat{g}_{4}}{\partial x_{1}} & \frac{\partial \hat{g}_{4}}{\partial x_{2}} & \frac{\partial \hat{g}_{4}}{\partial x_{3}} & \frac{\partial \hat{g}_{4}}{\partial x_{4}} & \frac{\partial \hat{g}_{4}}{\partial x_{5}} \\
\frac{\partial \hat{g}_{5}}{\partial x_{1}} & \frac{\partial \hat{g}_{5}}{\partial x_{2}} & \frac{\partial \hat{g}_{5}}{\partial x_{3}} & \frac{\partial \hat{g}_{5}}{\partial x_{4}} & \frac{\partial \hat{g}_{5}}{\partial x_{5}} \\
& & & &
\end{array}\right) \\
& =\left(\begin{array}{lllll}
\beta_{1} I+\mu & \beta_{1} E & 0 & -\beta_{9} & 0 \\
\beta_{10} I & \beta_{10} E+\beta_{8}+\beta_{6}+\beta_{2}+\mu & 0 & 0 & 0 \\
0 & -\beta_{2} & \beta_{3}+\beta_{5}+\mu & -\beta_{4} & 0 \\
0 & -\beta_{8} & -\beta_{3} & \beta_{9}+\beta_{4}+\mu & 0 \\
\beta_{7} D & -\beta_{6} & -\beta_{5} & 0 & \beta_{7} E
\end{array}\right)
\end{aligned}
$$

\section{Lemma 3.9}

(i) $\operatorname{det} \mathbb{V}=\beta_{7} E\left[\left(\beta_{1} I+\mu\right) \alpha\left(\beta \gamma-\beta_{3} \beta_{4}\right)-\beta_{10} I \beta_{1} E\left(\beta \gamma-\beta_{3} \beta_{4}\right)+\beta_{10} I \beta_{9}\left(\beta_{2} \beta_{3}+\beta \beta_{8}\right)\right]$

where

$\alpha=\beta_{10} E+\beta_{8}+\beta_{6}+\beta_{2}+\mu$

$\beta=\beta_{3}+\beta_{5}+\mu$

$\gamma=\beta_{9}+\beta_{4}+\mu$

(ii) $\mathbb{V}^{-1}=\frac{1}{\operatorname{det} \mathbb{V}}(-1)^{i+j} \mathbb{M}^{T}$

where $\mathbb{M}^{T}$ is the transpose of matrix of minor $\left(M_{i j}\right)$ of $\mathbb{V} \quad 1 \leq i, j \leq 5$

As the form of the matrix $\mathbb{F}$ is simple $\mathbb{F}=\left(\begin{array}{lllll}\beta_{7} D+\beta_{10} I & \beta_{10} E & 0 & 0 & \beta_{7} E \\ \beta_{1} I & \beta_{1} E & 0 & 0 & 0 \\ 0 & 0 & 0 & 0 & 0 \\ 0 & 0 & 0 & 0 & 0 \\ 0 & 0 & 0 & 0 & 0\end{array}\right)$ then the matrix $\mathbb{F V}^{-1}$ has the

Following form:

$\mathrm{FV}^{-1}=\frac{1}{\operatorname{det} \mathbb{V}}(-1)^{i+j}\left(\begin{array}{ll}A & B \\ C & D\end{array}\right)$ where $A$ is $2 \times 2$ matrix , $B$ is $2 \times 3$ matrix,$C=0$ is $3 \times 2$ matrix and $D=0$ is $3 \times 3$ matrix.

Lemma 3.10 


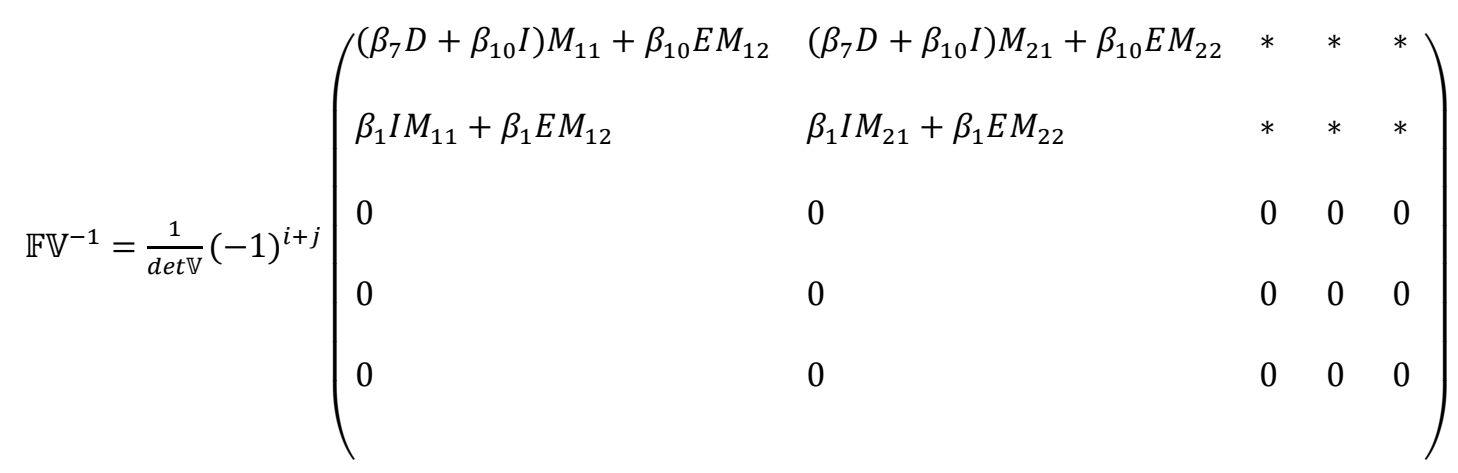

Where:

$$
\begin{aligned}
& M_{11}=\alpha \beta_{7} E\left(\beta \gamma-\beta_{3} \beta_{4}\right) \\
& \left.M_{12}=\beta_{1} E\left(\beta \gamma-\beta_{3} \beta_{4}\right)-\beta_{9}\left(\beta_{2} \beta_{3}\right)+\beta \beta_{8}\right) \\
& \left.M_{21}=\beta_{1} E\left(\beta \gamma-\beta_{3} \beta_{4}\right)-\beta_{9}\left(\beta_{2} \beta_{3}\right)+\beta \beta_{8}\right) \\
& \text { and } \\
& M_{22}=\beta_{7} E\left(\beta_{1}+\mu\right)\left(\beta \gamma-\beta_{3} \beta_{4}\right)
\end{aligned}
$$

In order to simplify the notations and avoid lengthy expressions, we define the parameters:

$a=\frac{1}{\operatorname{det} \mathbb{V}}\left[\left(\beta_{7} D+\beta_{10} I\right) M_{11}+\beta_{10} E M_{12}\right]$,

$b=\frac{-1}{\operatorname{det} \mathbb{V}}\left[\left(\beta_{7} D+\beta_{10} I\right) M_{21}+\beta_{10} E M_{22}\right]$,

$c=\frac{-1}{\operatorname{det} \mathbb{V}}\left[\beta_{1} I M_{11}+\beta_{1} E M_{12}\right]$,

And;

$d=\frac{1}{\operatorname{det} \mathbb{V}}\left[\beta_{1} I M_{21}+\beta_{1} E M_{22}\right]$

then the eigenvalues of $\mathbb{F V}^{-1}$ are $\lambda_{i} ; 1 \leq i \leq 5$ where $\lambda_{1}$ and $\lambda_{2}$ are the zeros of $(a-\lambda)(d-\lambda)-b c=\lambda^{2}-(a+d) \lambda+(a d-b c)=0$

And;

$\lambda_{3}=\lambda_{4}=\lambda_{5}=0$.

Consequently :

\section{Lemma 3.11}

$\mathcal{R}_{0}=\frac{a+d+\sqrt{\Delta}}{2}$ where $\Delta=(a+d)^{2}-4(a d-b c)$

In next section, we apply the corollary 2.11 to stability of Covid-19 system.

\section{Determination of Equilibrium Points}

\section{Theorem 4.1}

If the control reproduction number $\mathcal{R}_{0}$ is is less than 1 , model (covid-19) has a unique equilibrium: the disease-free equilibrium (DFE) $P_{0}=\left(\frac{B}{\mu}, 0,0,0,0\right)$.

Conversely, if $\mathcal{R}_{0}>1$, model (covid-19) has two equilibria: the DFE and a unique endemic equilibrium $P^{*}=$ $\left(E^{*}, I^{*}, C^{*}, H^{*}, D^{*}\right)=\left(E^{*}, \hat{\alpha} H^{*}, \hat{\beta} H^{*}, H^{*}, \hat{\gamma} H^{*}\right)$ where $H^{*}=\frac{B-\mu E^{*}}{\left[\left(\beta_{1}-\beta_{10}\right) \hat{\alpha}-\beta_{7} \hat{\gamma}\right] E^{*}-\beta_{9}}$ and $E^{*}=\frac{\mu+\beta_{2}+\beta_{6}+\beta_{8}}{\beta_{1}-\beta_{10}}=\frac{\alpha}{\beta_{1}-\beta_{10}}$

with

$\alpha=\beta_{2}+\beta_{6}+\beta_{8}+\mu$

$\hat{\alpha}=\frac{\beta_{3} \beta_{4}+\left(\beta_{4}+\beta_{3}+\mu\right)\left(\beta_{5}+\beta_{3}+\mu\right)}{\beta_{2} \beta_{3}+\beta_{8}\left(\beta_{5}+\beta_{3}+\mu\right)} ;$ 
$\hat{\beta}=\frac{\beta_{8} \beta_{4}+\beta_{2}\left(\beta_{4}+\beta_{9}+\mu\right)}{\beta_{2} \beta_{3}+\beta_{8}\left(\beta_{5}+\beta_{3}+\mu\right)}$

and

$\hat{\gamma}=\frac{\beta_{6}}{\beta_{7} E^{*}} \hat{\alpha}+\frac{\beta_{5}}{\beta_{7} E^{*}} \hat{\beta}$.

Proof

$\left\{\begin{array}{l}0=B-\beta_{1} E I+\beta_{7} E D+\beta_{9} H+\beta_{10} E I-\mu E \\ 0=\beta_{1} E I-\beta_{2} I-\beta_{6} I-\beta_{8} I-\beta_{10} E I-\mu I \\ 0=\beta_{2} I-\beta_{5} C-\beta_{3} C+\beta_{4} H-\mu C \\ 0=\beta_{3} C-\beta_{4} H+\beta_{8} I-\beta_{9} H-\mu H \\ 0=\beta_{5} C+\beta_{6} I-\beta_{7} D E\end{array}\right.$

(i) We observe that $P_{0}=\left(\frac{B}{\mu}, 0,0,0,0\right)$ is an equilibrium point which is called disease free equilibrium point.

(ii) A second equilibrium point $P^{*}$ is given by $P^{*}=\left(E^{*}, I^{*}, C^{*}, H^{*}, D^{*}\right)=\left(E^{*}, \hat{\alpha} H^{*}, \hat{\beta} H^{*}, H^{*}, \hat{\gamma} H^{*}\right)$ where $H^{*}=\frac{B-\mu E^{*}}{\left[\left(\beta_{1}-\beta_{10}\right) \widehat{\alpha}-\beta_{7} \widehat{\gamma}\right] E^{*}-\beta_{9}}$ and $E^{*}=\frac{\mu+\beta_{2}+\beta_{6}+\beta_{8}}{\beta_{1}-\beta_{10}}$ which is called Endemic equilibrium point.

In fact, If $I \neq 0$ then from equation (2), we deduce that $E^{*}=\frac{\mu+\beta_{2}+\beta_{6}+\beta_{8}}{\beta_{1}-\beta_{10}}$.

Writing the equations (3) and (4) in the following form

$$
\left\{\begin{array}{l}
\beta_{2} I-\left(\beta_{5}+\beta_{3}+\mu\right) C=-\beta_{4} H \\
\beta_{8} I+\beta_{3} C=\left(\beta_{4}+\beta_{9}+\mu\right) H
\end{array}\right.
$$

to deduce that

$I=\frac{\left[\beta_{3} \beta_{4}+\left(\beta_{4}+\beta_{3}+\mu\right)\left(\beta_{5}+\beta_{3}+\mu\right)\right] H}{\beta_{2} \beta_{3}+\beta_{8}\left(\beta_{5}+\beta_{3}+\mu\right)}=\hat{\alpha} H$,

where $\hat{\alpha}=\frac{\beta_{3} \beta_{4}+\left(\beta_{4}+\beta_{3}+\mu\right)\left(\beta_{5}+\beta_{3}+\mu\right)}{\beta_{2} \beta_{3}+\beta_{8}\left(\beta_{5}+\beta_{3}+\mu\right)}$

and

$C=\frac{\left[\beta_{8} \beta_{4}+\beta_{2}\left(\beta_{4}+\beta_{9}+\mu\right)\right] H}{\beta_{2} \beta_{3}+\beta_{8}\left(\beta_{5}+\beta_{3}+\mu\right)}=\hat{\beta} H$

where $\hat{\beta}=\frac{\beta_{8} \beta_{4}+\beta_{2}\left(\beta_{4}+\beta_{9}+\mu\right)}{\beta_{2} \beta_{3}+\beta_{8}\left(\beta_{5}+\beta_{3}+\mu\right)}$

it follows from equation (5) that $D=\frac{\beta_{6}}{\beta_{7} E} I+\frac{\beta_{5}}{\beta_{7} E} C=\left[\frac{\beta_{6}}{\beta_{7} E} \hat{\alpha}+\frac{\beta_{5}}{\beta_{7} E} \hat{\beta}\right] H=\hat{\gamma} H$

where $\hat{\gamma}=\frac{\beta_{6}}{\beta_{7} E} \hat{\alpha}+\frac{\beta_{5}}{\beta_{7} E} \hat{\beta}$.

and from equation (1), we deduce that $H=\frac{B-\mu E}{\left[\left(\beta_{1}-\beta_{10}\right) \widehat{\alpha}-\beta_{7} \widehat{\gamma}\right] E-\beta_{9}}$.

Then we get $P^{*}=\left(E^{*}, I^{*}, C^{*}, H^{*}, D^{*}\right)=\left(E^{*}, \hat{\alpha} H^{*}, \hat{\beta} H^{*}, H^{*}, \hat{\gamma} H^{*}\right)$

where $H^{*}=\frac{B-\mu E^{*}}{\left[\left(\beta_{1}-\beta_{10}\right) \widehat{\alpha}-\beta_{7} \widehat{\gamma}\right] E^{*}-\beta_{9}}$

and

$E^{*}=\frac{\mu+\beta_{2}+\beta_{6}+\beta_{8}}{\beta_{1}-\beta_{10}}$ which is called Endemic equilibrium point.

\section{Corollary 4.2}

If the parameters $\left(\beta_{1}, \beta_{2}, \ldots, \beta_{10}\right)$ satisfy one of the following conditions :

(i) $\beta_{1}<\beta_{10}$; 
(ii) $\left(\beta_{2}+\beta_{6}+\beta_{8}+\mu\right) \hat{\alpha}<\beta_{5} \hat{\beta}+\beta_{9}$.

Then the model (covid-19) has a unique equilibrium:

The disease-free equilibrium (DFE) $P_{0}=\left(\frac{B}{\mu}, 0,0,0,0\right)$.

\section{Definition 4.3}

The equilibrium $P^{*}=\left(E^{*}, I^{*}, C^{*}, H^{*}, D^{*}\right)$ is called feasible if its components are positive.

Thanks to van den Driessche and Watmough (2002) [8], the following result is straightforward.

\section{Theorem 4.4}

If $\mathcal{R}_{0}<1$, the DFE is locally asymptotically stable. If $\mathcal{R}_{0}>1$, the DFE is unstable.

The epidemiological interpretation of Theorem 4.4 is that, (covid-19) can be eliminated in the population when $\mathcal{R}_{0}<1$ if the initial conditions of the dynamical system (covid-19) are in the basin of attraction of the DFE $P_{0}$.

The theorem 4.4 shows also that, $\mathcal{R}_{0}$ is a threshold which can determine if the disease will be spread or not. Thus, reducing its value, is a means to mitigate or even eliminate the (covid-19) . It can be therefore important to determine among model parameters those who mostly influence its value.

Now let $e(t)=E(t)-E^{*}, i(t)=I(t)-I^{*}, c(t)=C(t)-C^{*}, h(t)=H(t)-H^{*}$ and $d(t)=D(t)-D^{*}$ then it is easy to verify that $e, i, c, h$ and $d$ satisfy the following system of differential equations:

$$
\left\{\begin{array}{l}
\frac{d e(t)}{d t}=\left[\left(\beta_{10}-\beta_{1}\right) I^{*}+\beta_{7} D^{*}-\mu\right] e(t)+\left(\beta_{10}-\beta_{1}\right) E^{*} i(t)+\beta_{7} E^{*} d(t)+\beta_{9} h(t)+\left(\beta_{10}-\beta_{1}\right) e(t) i(t)+\beta_{7} e(t) d(t) \\
\frac{d i(t)}{d t}=\left[\left(\beta_{1}-\beta_{10}\right) I^{*}+\beta_{7} D^{*}-\mu\right] e(t)+\left[\left(\beta_{1}-\beta_{10}\right) E^{*}-\left(\beta_{2}+\beta_{6}+\beta_{8}+\mu\right)\right] i(t)+\left(\beta_{1}-\beta_{10}\right) e(t) i(t) \\
\frac{d c(t)}{d t}=\beta_{2} i(t)-\left(\beta_{2}+\beta_{5}+\mu c(t)+\beta_{4} h(t)\right. \\
\frac{d h(t)}{d t}=\beta_{8} i(t)+\beta_{3} c(t)+\left(\beta_{8}-\beta_{4}-\beta_{9}-\mu h(t)\right. \\
\frac{d d(t)}{d t}=-\beta_{7} D^{*} e(t)+\beta_{6} i(t)+\beta_{5} c(t)-\beta_{7} E^{*} d(t)-\beta_{7} e(t) d(t)
\end{array}\right.
$$

with subject to the restriction $e+i+c+h+d \leq \frac{B}{\mu}-\left[E^{*}+(1+\alpha+\beta+\gamma) H^{*}\right]$.

the point $p^{*}=\left(e^{*}, i^{*}, c^{*}, h^{*}, d^{*}\right)=(0,0,0,0)$ is an equilibrium point of the system (4.1).

The jacobian matrix of the system (4.1) is given by:

$\mathbb{J}_{p^{*}}=\left(\begin{array}{ccccc}a_{11} & a_{12} & a_{13} & a_{14} & a_{15} \\ a_{21} & a_{22} & a_{23} & a_{24} & a_{25} \\ a_{31} & a_{32} & a_{33} & a_{34} & a_{35} \\ a_{41} & a_{42} & a_{43} & a_{44} & a_{45} \\ a_{51} & a_{52} & a_{53} & a_{54} & a_{55}\end{array}\right)$

where

- $a_{11}=\left(\beta_{10}-\beta_{1}\right) I^{*}+\beta_{7} D^{*}-\mu, a_{12}=\left(\beta_{10}-\beta_{1}\right) E^{*}, a_{13}=0, a_{14}=\beta_{9}, a_{15}=\beta_{7} E^{*}$

- $a_{21}=\left(\beta_{1}-\beta_{10}\right) I^{*}+\beta_{7} D^{*}-\mu, a_{22}=\left(\beta_{1}-\beta_{10}\right) E^{*}-\left(\beta_{2}+\beta_{6}+\beta_{8}+\mu\right), a_{23}=0, a_{24}=0, a_{25}=0$

- $a_{31}=0, a_{32}=\beta_{2}, a_{33}=-\left(\beta_{2}+\beta_{5}+\mu\right), a_{34}=\beta_{4}, a_{35}=0$

- $a_{41}=0, a_{42}=\beta_{8}, a_{43}=\beta_{3}, a_{44}=\beta_{8}-\beta_{4}-\beta_{9}-\mu, a_{45}=0$

- $a_{51}=-\beta_{7} D^{*}, a_{52}=\beta_{6}, a_{53}=\beta_{5}, a_{54}=0, a_{55}=-\beta_{7} E^{*}$ 


$$
\text { i.e. } \mathbb{J}_{p^{*}}=\left(\begin{array}{lllll}
a_{11} & a_{12} & 0 & a_{14} & a_{15} \\
a_{21} & a_{22} & 0 & 0 & 0 \\
0 & a_{32} & a_{33} & a_{34} & 0 \\
0 & a_{42} & a_{43} & a_{44} & 0 \\
a_{51} & a_{52} & a_{53} & 0 & a_{55}
\end{array}\right)
$$

In particular we deduce that

$$
\mathbb{J}_{p^{0}}=\left(\begin{array}{lllll}
-\mu & \left(\beta_{10}-\beta_{1}\right) E & 0 & \beta_{9} & \beta_{7} E \\
-\mu & \left(\beta_{1}-\beta_{10}\right) E-\left(\beta_{2}+\beta_{6}+\beta_{8}+\mu\right) & 0 & 0 & 0 \\
0 & \beta_{2} & -\left(\beta_{2}+\beta_{5}+\mu\right) & \beta_{4} \\
0 & \beta_{8} & \beta_{3} & \beta_{8}-\beta_{4}-\beta_{9}-\mu & 0 \\
0 & 0 & 0 & 0 & -\beta_{7} E
\end{array}\right)
$$

where $E=\frac{B}{\mu}$

Now we recall some technic calculations of determinant of a matrix in the following form :

\section{Lemma 4.5}

$$
\text { let } A=\left(\begin{array}{cccc}
a_{1,1} & a_{1,2} & a_{1,3} & a_{1,4} \\
a_{2,1} & a_{2,2} & a_{2,3} & a_{2,4} \\
a_{3,1} & a_{3,2} & a_{3,3} & a_{3,4} \\
a_{4,1} & a_{4,2} & a_{4,3} & a_{4,4}
\end{array}\right) \text { then we have }
$$$$
\operatorname{det}\left(\begin{array}{llll}
a_{1,1} & a_{1,2} & a_{1,3} & a_{1,4} \\
a_{2,1} & a_{2,2} & a_{2,3} & a_{2,4} \\
a_{3,1} & a_{3,2} & a_{3,3} & a_{3,4} \\
a_{4,1} & a_{4,2} & a_{4,3} & a_{4,4}
\end{array}\right)=\operatorname{det}\left(\begin{array}{cc}
a_{1,1} & a_{1,2} \\
a_{2,1} & a_{2,2}
\end{array}\right) \operatorname{det}\left(\begin{array}{ll}
a_{3,3} & a_{3,4} \\
a_{4,3} & a_{4,4}
\end{array}\right)-\operatorname{det}\left(\begin{array}{ll}
a_{1,1} & a_{1,2} \\
a_{3,1} & a_{3,2}
\end{array}\right) \operatorname{det}\left(\begin{array}{ll}
a_{2,3} & a_{2,4} \\
a_{4,3} & a_{4,4}
\end{array}\right)+
$$$$
\operatorname{det}\left(\begin{array}{cc}
a_{1,1} & a_{1,2} \\
& \\
a_{4,1} & a_{4,2}
\end{array}\right) \operatorname{det}\left(\begin{array}{ll}
a_{2,3} & a_{2,4} \\
& \\
a_{3,3} & a_{3,4}
\end{array}\right)+\operatorname{det}\left(\begin{array}{ll}
a_{2,1} & a_{2,2} \\
& \\
a_{3,1} & a_{3,2}
\end{array}\right) \operatorname{det}\left(\begin{array}{ll}
a_{1,3} & a_{1,4} \\
& \\
a_{4,3} & a_{4,4}
\end{array}\right)-\operatorname{det}\left(\begin{array}{ll}
a_{2,1} & a_{2,2} \\
& \\
a_{4,1} & a_{4,2}
\end{array}\right) \operatorname{det}\left(\begin{array}{cc}
a_{1,3} & a_{1,4} \\
a_{3,3} & a_{3,4}
\end{array}\right)+
$$
$\operatorname{det}\left(\begin{array}{ll}a_{3,1} & a_{3,2} \\ a_{4,1} & a_{4,2}\end{array}\right) \operatorname{det}\left(\begin{array}{ll}a_{1,3} & a_{1,4} \\ a_{2,3} & a_{2,4}\end{array}\right)$

i.e.

$$
\begin{aligned}
\left|\begin{array}{llll}
a_{11} & a_{12} & a_{13} & a_{14} \\
a_{21} & a_{22} & a_{23} & a_{24} \\
a_{31} & a_{32} & a_{33} & a_{34} \\
a_{41} & a_{42} & a_{43} & a_{14}
\end{array}\right| & =\left|\begin{array}{ll}
a_{11} & a_{12} \\
a_{21} & a_{22}
\end{array}\right|\left|\begin{array}{ll}
a_{33} & a_{34} \\
a_{43} & a_{44}
\end{array}\right|-\left|\begin{array}{ll}
a_{11} & a_{12} \\
a_{31} & a_{32}
\end{array}\right|\left|\begin{array}{ll}
a_{23} & a_{24} \\
a_{43} & a_{44}
\end{array}\right|+\left|\begin{array}{ll}
a_{11} & a_{12} \\
a_{41} & a_{42}
\end{array}\right|\left|\begin{array}{ll}
a_{23} & a_{24} \\
a_{33} & a_{34}
\end{array}\right| \\
& +\left|\begin{array}{ll}
a_{21} & a_{22} \\
a_{31} & a_{32}
\end{array}\right|\left|\begin{array}{cc}
a_{13} & a_{14} \\
a_{43} & a_{44}
\end{array}\right|-\left|\begin{array}{ll}
a_{21} & a_{22} \\
a_{41} & a_{42}
\end{array}\right|\left|\begin{array}{ll}
a_{13} & a_{14} \\
a_{33} & a_{34}
\end{array}\right|+\left|\begin{array}{ll}
a_{31} & a_{32} \\
a_{41} & a_{42}
\end{array}\right|\left|\begin{array}{ll}
a_{13} & a_{14} \\
a_{23} & a_{24}
\end{array}\right|
\end{aligned}
$$


In particular we have:

$\operatorname{det}\left(\begin{array}{cccc}a_{1,1} & a_{1,2} & 0 & a_{1,4} \\ a_{2,1} & a_{2,2} & 0 & 0 \\ 0 & a_{3,2} & a_{3,3} & 0 \\ 0 & a_{4,2} & a_{4,3} & a_{4,4}\end{array}\right)=$

$\operatorname{det}\left(\begin{array}{ll}a_{1,1} & a_{1,2} \\ a_{2,1} & a_{2,2}\end{array}\right) \operatorname{det}\left(\begin{array}{cc}a_{3,3} & 0 \\ a_{4,3} & a_{4,4}\end{array}\right)-\operatorname{det}\left(\begin{array}{cc}a_{1,1} & a_{1,2} \\ 0 & a_{3,2}\end{array}\right) \operatorname{det}\left(\begin{array}{cc}0 & 0 \\ & \\ a_{4,3} & a_{4,4}\end{array}\right)+\operatorname{det}\left(\begin{array}{cc}a_{1,1} & a_{1,2} \\ 0 & a_{4,2}\end{array}\right) \operatorname{det}\left(\begin{array}{c}0 \\ a_{3,3} \\ 0\end{array}\right)+$ $\operatorname{det}\left(\begin{array}{cc}a_{2,1} & a_{2,2} \\ 0 & a_{3,2}\end{array}\right) \operatorname{det}\left(\begin{array}{cc}0 & a_{1,4} \\ a_{4,3} & a_{4,4}\end{array}\right)-\operatorname{det}\left(\begin{array}{cc}a_{2,1} & a_{2,2} \\ 0 & a_{4,2}\end{array}\right) \operatorname{det}\left(\begin{array}{cc}0 & a_{1,4} \\ a_{3,3} & 0\end{array}\right)+\operatorname{det}\left(\begin{array}{cc}0 & a_{3,2} \\ 0 & a_{4,2}\end{array}\right) \operatorname{det}\left(\begin{array}{cc}0 & a_{1,4} \\ 0 & 0\end{array}\right)$

$=a_{11} a_{22} a_{33} a_{44}+a_{21} a_{42} a_{14} a_{33}-\left(a_{21} a_{12} a_{33} a_{44}+a_{21} a_{32} a_{14} a_{43}\right)$

\section{Corollary 4.6}

Let $\mathbb{J}_{p^{0}}=\left(\begin{array}{lllll}-\mu & \left(\beta_{10}-\beta_{1}\right) E^{*} & 0 & \beta_{9} & \beta_{7} E^{*} \\ -\mu & \left(\beta_{1}-\beta_{10}\right) E^{*}-\left(\beta_{2}+\beta_{6}+\beta_{8}+\mu\right) & 0 & 0 & 0 \\ 0 & \beta_{2} & -\left(\beta_{2}+\beta_{5}+\mu\right) & \beta_{4} & 0 \\ 0 & \beta_{8} & \beta_{3} & \beta_{8}-\beta_{4}-\beta_{9}-\mu & 0 \\ 0 & 0 & 0 & 0 & -\beta_{7} E^{*}\end{array}\right)$

where $E^{*}=\frac{B}{\mu}$

Then we have

(i) $\operatorname{det} \mathbb{1}_{p^{0}}=-\beta_{7} E^{*} \operatorname{det}\left(\begin{array}{llll}-\mu & \left(\beta_{10}-\beta_{1}\right) E^{*} & 0 & \beta_{9} \\ -\mu & \left(\beta_{1}-\beta_{10}\right) E^{*}-\left(\beta_{2}+\beta_{6}+\beta_{8}+\mu\right) & 0 & 0 \\ 0 & \beta_{2} & -\left(\beta_{2}+\beta_{5}+\mu\right) & \beta_{4} \\ 0 & \beta_{8} & \beta_{3} & \beta_{8}-\beta_{4}-\beta_{9}-\mu\end{array}\right)$

$=-\mu \beta_{7} E^{*}\left\{\beta\left(\beta_{8}-\gamma\right)\left(a E^{*}-\alpha\right)+\beta \beta_{8} \beta_{9}+\beta\left(\beta_{8} a E^{*}-\gamma\right)+\beta_{2} \beta_{3} \beta_{9}\right\}$

$=-\mu \beta_{7} \beta E^{*}\left(2 \beta_{8} a E^{*}+\alpha \gamma+\beta_{8} \beta_{9}-\beta_{8} \alpha\right)$.

Where $a=\beta_{1}-\beta_{10}, \alpha=\beta_{2}+\beta_{6}+\beta_{8}+\mu, \beta=\beta_{2}+\beta_{5}+\mu$ and $\gamma=\beta_{4}+\beta_{9}+\mu$

(ii) Let $\beta_{10}<\beta_{1}$ and $2 \beta_{8} a E^{*}+\alpha \gamma+\beta_{8} \beta_{9}>\beta_{8} \alpha$ then $\operatorname{det} \mathbb{J}_{p^{0}}<0$.

(iii) Under the conditions of (ii) we observe that a assymption of Li-Wang criterion is satisfied.

Now, if $\beta_{10}<\beta_{1}$, we write $\mathbb{I}_{p^{0}}$ in the following form

$\mathbb{J}_{p^{0}}=\mathbb{M}-\mathbb{E}$ where 


$$
\mathbb{M}=\left(\begin{array}{lllll}
0 & 0 & 0 & \beta_{9} & \beta_{7} E^{*} \\
0 & \left(\beta_{1}-\beta_{10}\right) E^{*} & 0 & 0 & 0 \\
0 & \beta_{2} & 0 & \beta_{4} & 0 \\
0 & \beta_{8} & \beta_{3} & \beta_{8} & 0 \\
0 & 0 & 0 & 0 & 0
\end{array}\right) \text { and } \mathbb{E}=\left(\begin{array}{lllll}
\mu & \left(\beta_{1}-\beta_{10}\right) E^{*} & 0 & 0 & 0 \\
\mu & \alpha & 0 & 0 & 0 \\
0 & 0 & \beta & 0 & 0 \\
0 & 0 & 0 & \gamma & 0 \\
0 & 0 & 0 & 0 & \beta_{7} E^{*}
\end{array}\right)
$$

Then if $E^{*} \neq \frac{\alpha}{\beta_{1}-\beta_{10}}$ we deduce that:

$\mathbb{E}^{-1}=\left(\begin{array}{lllll}\left.\frac{\alpha}{\mu(\alpha-\text { reda }}\right) & \frac{-a}{\mu(\alpha-a)} & 0 & 0 & 0 \\ \frac{-1}{\alpha-a} & \frac{1}{\alpha-a} & 0 & 0 & 0 \\ 0 & 0 & \frac{1}{\beta} & 0 & 0 \\ 0 & 0 & 0 & \frac{1}{\gamma} & 0 \\ 0 & 0 & 0 & 0 & \frac{1}{\beta_{7} E^{*}}\end{array}\right)$

where $a=\left(\beta_{1}-\beta_{10}\right) E^{*}$

and

$$
\mathbb{M E}^{-1}=\left(\begin{array}{lllll}
0 & 0 & 0 & \frac{\beta_{9}}{\gamma} & 1 \\
\text { au } & \text { av } & 0 & 0 & 0 \\
\beta_{2} u & \beta_{2} v & 0 & \frac{\beta_{4}}{\gamma} & 0 \\
\beta_{8} u & \beta_{8} v & \frac{\beta_{3}}{\beta} & \frac{\beta_{8}}{\gamma} & 0 \\
0 & 0 & 0 & 0 & 0
\end{array}\right) \text { where } u=\frac{-1}{\alpha-a} \text { and } v=\frac{1}{\alpha-a}
$$

and

$$
\begin{aligned}
& \chi(\lambda):=\operatorname{det}\left(\mathbb{M E}^{-1} \lambda I\right)=\left|\begin{array}{lllll}
-\lambda & 0 & 0 & \frac{\beta_{9}}{\gamma} & 1 \\
a u & a v-\lambda & 0 & 0 & 0 \\
\beta_{2} u & \beta_{2} v & -\lambda & \frac{\beta_{4}}{\gamma} & 0 \\
\beta_{8} u & \beta_{8} v & \frac{\beta_{3}}{\beta} & \frac{\beta_{8}}{\gamma}-\lambda & 0 \\
0 & 0 & 0 & 0 & -\lambda
\end{array}\right|=-\lambda\left|\begin{array}{llll}
-\lambda & 0 & 0 & \frac{\beta_{9}}{\gamma} \\
a u & a v-\lambda & 0 & 0 \\
\beta_{2} u & \beta_{2} v & -\lambda & \frac{\beta_{4}}{\gamma} \\
\beta_{8} u & \beta_{8} v & \frac{\beta_{3}}{\beta} & \frac{\beta_{8}}{\gamma}-\lambda
\end{array}\right| \\
& =\lambda^{2}\left|\begin{array}{lll}
a v-\lambda & 0 & 0 \\
\beta_{2} v & -\lambda & \frac{\beta_{4}}{\gamma} \\
\beta_{8} v & \frac{\beta_{3}}{\beta} & \frac{\beta_{8}}{\gamma}-\lambda
\end{array}\right|+\frac{\beta_{9}}{\gamma} \lambda\left|\begin{array}{lll}
a u & a v-\lambda & 0 \\
\beta_{2} u & \beta_{2} v & -\lambda \\
\beta_{8} u & \beta_{8} v & \frac{\beta_{3}}{\beta}
\end{array}\right| \\
& =\lambda^{2}(a v-\lambda)\left|\begin{array}{ll}
-\lambda & \frac{\beta_{4}}{\gamma} \\
\frac{\beta_{3}}{\beta} & \frac{\beta_{8}}{\gamma}-\lambda
\end{array}\right|+\frac{\beta_{9}}{\gamma} \lambda a u\left|\begin{array}{ll}
\beta_{2} v & -\lambda \\
\beta_{8} v & \frac{\beta_{3}}{\beta} \\
-1
\end{array}\right|-\frac{\beta_{9}}{\gamma} \lambda(a v-\lambda)\left|\begin{array}{ll}
\beta_{2} u & -\lambda \\
\beta_{8} u & \frac{\beta_{3}}{\beta}
\end{array}\right| \\
& =-\lambda^{2}(a v-\lambda)\left[\lambda\left(\frac{\beta_{8}}{\gamma}-\lambda\right)+\frac{\beta_{3} \beta_{4}}{\beta \gamma}\right]+\frac{\beta_{9}}{\gamma} \lambda a u v\left(\frac{\beta_{2} \beta_{3}}{\beta}+\beta_{8} \lambda\right)-\frac{\beta_{9}}{\gamma} \lambda(a v-\lambda) u\left(\frac{\beta_{2} \beta_{3}}{\beta}+\beta_{8} \lambda\right)
\end{aligned}
$$




$$
\begin{aligned}
& =-\lambda^{2}(a v-\lambda)\left(-\lambda^{2}+\frac{\beta_{8}}{\gamma} \lambda+\frac{\beta_{3} \beta_{4}}{\beta \gamma}\right)+\frac{\beta_{9} \beta_{8}}{\gamma} a u v \lambda^{2}+\frac{\beta_{9} \beta_{8}}{\gamma} u \lambda^{2} . \\
& =-\lambda^{2}\left(-a v \lambda^{2}+\frac{\beta_{8}}{\gamma} a v \lambda+\frac{\beta_{3} \beta_{4}}{\beta \gamma} a v+\lambda^{3}-\frac{\beta_{8}}{\gamma} \lambda^{2}-\frac{\beta_{3} \beta_{4}}{\beta \gamma} \lambda\right)+\frac{\beta_{9} \beta_{8}}{\gamma} a u v \lambda^{2}+\frac{\beta_{9} \beta_{8}}{\gamma} u \lambda^{2} \\
& =-\lambda^{2}\left[\lambda^{3}+\left(\frac{\beta_{9} \beta_{8}}{\gamma} u(a v+1)-a v-\frac{\beta_{8}}{\gamma}\right) \lambda^{2}+\left(\frac{\beta_{8}}{\gamma} a v-\frac{\beta_{3} \beta_{4}}{\beta \gamma}\right) \lambda+\frac{\beta_{3} \beta_{4}}{\beta \gamma} a v\right] . \\
& \text { Setting } a_{1}=\frac{\beta_{9} \beta_{8}}{\gamma} u(a v+1)-a v-\frac{\beta_{8}}{\gamma}, a_{2}=\frac{\beta_{8}}{\gamma} a v-\frac{\beta_{3} \beta_{4}}{\beta \gamma}, a_{3}=\frac{\beta_{3} \beta_{4}}{\beta \gamma} a v,
\end{aligned}
$$

and

$\chi(\lambda)=\lambda^{3}+a_{1} \lambda^{2}+a_{2} \lambda+a_{3}$. If $\lambda_{1}, \lambda_{1}$ and $\lambda_{3}$ are the zeros of $\chi(\lambda)=0$

Then we have

- $\lambda_{1}+\lambda_{2}+\lambda_{3}=-a_{1}$

- $\lambda_{1} \lambda_{2}+\lambda_{1} \lambda_{3}+\lambda_{2} \lambda_{3}=a_{2}$

- $\lambda_{1} \lambda_{2} \lambda_{3}=-a_{3}$.

and

\section{Proposition 4.7}

Let $\chi(\lambda)=\lambda^{3}+a_{1} \lambda^{2}+a_{2} \lambda+a_{3}$, so that $\chi$ is uniformly asymptotically stable (uas), it is necessary that it suffices that $\Delta_{1}=a_{1}>0, \Delta_{2}=a_{1} a_{2}-a_{3}>0$ and $\Delta_{3}=a_{3} \Delta_{2}>0$.

A necessary condition for all the roots of the characteristic polynomial to admit a negative real part, all the coefficients must be positive, that is to say: $a_{1}>0, a_{2}>0, \ldots, a_{3}>0$.

- As $\frac{a_{1}=\beta_{9} \beta_{8}}{\gamma} u(a v+1)-a v-\frac{\beta_{8}}{\gamma} \leq 0$ then we can not apply this above proposition for $\chi(\lambda)$.

Now, if we consider the discriminant of $\chi$ which is given by :

$\Delta_{\chi}=a_{1}^{2} a_{2}^{2}+18 a_{1} a_{2} a_{3}-27 a_{3}^{2}-4 a_{2}^{3}-4 a_{1}^{3} a_{3}$.

we observe that :

$\bullet_{1}$ If $\Delta_{\chi}>0,3$ different real roots of the equation $\chi(\lambda)=0$.

$\bullet_{2}$ If $\Delta_{\chi}=0$, one double or triple root of the equation $\chi(\lambda)=0$.

- 3 If $\Delta_{\chi}<0$, one real root and two complex roots of the equation $\chi(\lambda)=0$.

${ }_{4}$ if $\Delta_{\chi}>0$, then a necessary and sufficient condition for an equilibrium point to be locally asymptotically stable is $a_{1}>0, a_{3}>0, a_{1} a_{2}-a_{3}>0$.

$\bullet_{5}$ if $\Delta_{\chi}<0, a_{1}<0, a_{2}<0$, then all roots of $\chi(\lambda)=0$ satisfy the condition $|\arg (\lambda)|<\frac{\pi}{2}$.

${ }_{6}{ }_{6}$ if $\Delta_{\chi}>0, a_{1}>0, a_{2}>0, a_{1} a_{2}-a_{3}=0$, then an equilibrium point is locally asymptotically stable.

- 7 A necessary condition for an equilibrium point to be locally asymptotically stable is $a_{3}>0$.

$\bullet_{8}$ if the conditions $\Delta_{\chi}<0, a_{1}>0, a_{2}>0, a_{1} a_{2}-a_{3}=0$ are satisfied, then an equilibrium point is not locally asymptotically stable.

We remark that $\bullet_{4}, \bullet_{6}$ and $\bullet_{4}$ are not satisfy by the coefficients of $\chi(\lambda)$. So we have to solve the cubic equation $\chi(\lambda)=0$ by the Cardan's method which is ingenious and effective, but quite non-intuitive.

Theorem 4.8 (solutions of cubic equation)

Let $P$ the general cubic equation:

$a x^{3}+b x^{2}+c x+d=0 ; a \neq 0$

Then $P$ has solutions:

$x_{1}=S+T-\frac{b}{3 a}$

$x_{2}=-\frac{S+T}{2}-\frac{b}{3 a}+i \sqrt{3}(S-T)$ 
$x_{3}=-\frac{S+T}{2}-\frac{b}{3 a}-i \sqrt{3}(S-T)$

where

$S=\sqrt[3]{R+\sqrt{R^{2}+Q^{3}}}$

$T=\sqrt[3]{R-\sqrt{R^{2}+Q^{3}}}$

and

$Q=\frac{3 c-b^{2}}{9 a^{2}}$

$R=\frac{9 a b c-27 a^{2} d-2 b^{3}}{54 a^{3}}$

The expression $\Delta=Q^{3}+R^{2}$ is called the discriminant of the equation

See for example Nickalls (1993) [57] for a brief description of Cardan's method.

- Substantial technical difficulties for explicit expression of $\mathbb{J}_{p^{*}}^{[2]}$

In Appendix of Li-Wang [2], we found that for $n=2,3$, and 4, an explicit expression of second additive compound matrices $A^{[2]}$ of $n \times n$ matrices $A=\left(a_{i j}\right)_{1 \leq i, j \leq n}$ which are given respectively by:

- $n=2: A^{[2]}=a_{11}+a_{22}=\operatorname{tr}(A)$

- $n=3: A^{[2]}=\left(\begin{array}{lll}a_{11}+a_{22} & a_{23} & -a_{13} \\ a_{32} & a_{11}+a_{33} & a_{12} \\ -a_{31} & a_{21} & a_{22}+a_{33}\end{array}\right)$

In the same way that the section 5 of Li-Wang [2] where they have studied the stability of an epidemic model of SEIR type, we apply their criterion to the following epidemic model:

$\left\{\begin{array}{l}\frac{d S}{d t}=\Lambda-\left(\beta_{1} I_{1}+\beta_{2} I_{2}\right) S-\mu S \\ \frac{d I_{1}}{d t}=\left(\beta_{1} I_{1}+\beta_{2} I_{2}\right) S-(\mu+\gamma) I_{1} \\ \frac{d I_{2}}{d t}=\gamma I_{1}-(\mu+d) I_{2}\end{array}\right.$

where $\left(\Lambda, \beta_{1},\left(\beta_{2}, \mu, \gamma, d\right)\right.$ are given parameters.

- Determination of equilibrium points of the system ( $\left.{ }^{*}{ }_{1}\right)$ and calculation of basic reproduction number $\mathcal{R}_{0}$

Let $E=\left(S, I_{1}, I_{2}\right)$ then the Jacobian matrix of above system is :

$\mathbb{J}_{E}=\left(\begin{array}{lll}-\left(\beta_{1} I_{1}+\beta_{2} I_{2}+\mu\right) & -\beta_{1} S & -\beta_{2} S \\ \beta_{1} I_{1}+\beta_{2} I_{2} & \beta_{1} S-\mu-\gamma & \beta_{2} S \\ 0 & \gamma & -\mu-d\end{array}\right)$

Now, we consider the following equations:

$\left\{\begin{array}{l}\frac{d S}{d t}=\Lambda-\left(\beta_{1} I_{1}+\beta_{2} I_{2}\right) S-\mu S=0 \\ \frac{d I_{1}}{d t}=\left(\beta_{1} I_{1}+\beta_{2} I_{2}\right) S-(\mu+\gamma) I_{1}=0 \\ \frac{d I_{2}}{d t}=\gamma I_{1}-(\mu+d) I_{2}=0\end{array}\right.$ 
then we observe that $E^{0}=\left(\frac{\Lambda}{\mu}, 0,0\right)$ is a trivial equilibrium point of $\left(*_{3}\right)$ (Disease free equilibrium point) and so

$$
\mathbb{J}_{E^{0}}=\left(\begin{array}{lll}
-\mu & -\frac{\beta_{1} \Lambda}{\mu} & -\frac{\beta_{2} \Lambda}{\mu} \\
0 & \frac{\beta_{1} \Lambda}{\mu}-\mu-\gamma & \frac{\beta_{2} \Lambda}{\mu} \\
0 & \gamma & -\mu-d
\end{array}\right)
$$

By using the next generation matrix method, the basic reproduction number $\mathcal{R}_{0}$ is obtained as the spectral radius of matrix $\left(-\mathbb{F V}^{-1}\right)$ at disease free equilibrium point where $\mathbb{F}$ and $\mathbb{V}$ are as below :

$$
\begin{aligned}
& \mathbb{F}=\left(\begin{array}{cc}
\frac{\beta_{1} \Lambda}{\mu} & \frac{\beta_{2} \Lambda}{\mu} \\
0 & 0
\end{array}\right), \mathbb{V}=\left(\begin{array}{cc}
-\mu-\gamma & 0 \\
\gamma & -\mu-d
\end{array}\right), \mathbb{V}^{-1}=\left(\begin{array}{ll}
-\frac{1}{\mu+\gamma} & 0 \\
-\frac{\gamma}{(\mu+d)(\mu+\gamma)} & -\frac{1}{\mu+d}
\end{array}\right) \\
& \text { and }-\mathbb{F V}^{-1}=\Lambda\left(\begin{array}{ll}
\frac{\beta_{1}(\mu+d)+\beta_{2} \gamma}{\mu(\mu+d)(\mu+\gamma)} & \frac{\beta_{2}}{\mu(\mu+d)} \\
0 & 0
\end{array}\right)
\end{aligned}
$$

It follows that :

$\mathcal{R}_{0}=\Lambda \frac{\beta_{1}(\mu+d)+\beta_{2} \gamma}{\mu(\mu+d)(\mu+\gamma)}$

Evolution of $\mathcal{R}_{0}$ with respect $\mu$

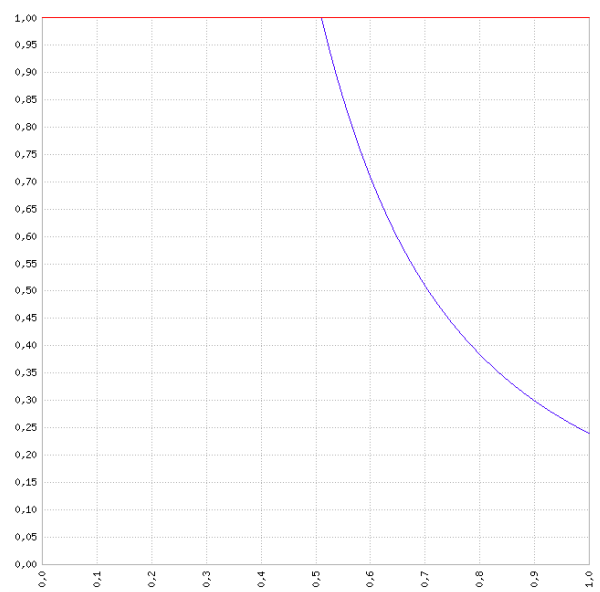

where $\beta_{1}=0,3, \beta_{2}=0,8, \gamma=0,1, \Lambda=0,7$ and $d=0,04$

$\mathbb{J}_{E^{0}}^{[2]}=\left(\begin{array}{lll}\frac{\beta_{1} \Lambda}{\mu}-2 \mu-\gamma & \frac{\beta_{2} \Lambda}{\mu} & \frac{\beta_{2} \Lambda}{\mu} \\ \gamma & -2 \mu-d & -\frac{\beta_{1} \Lambda}{\mu} \\ 0 & 0 & -2 \mu-\gamma-d\end{array}\right)$

Now let $I_{1} \neq 0$ then from (3) we deduce that :

$I_{2}=\delta I_{1} ; \delta=\frac{\gamma}{\mu+d}$

From $\left({ }^{*}\right)$ and $(2)$ we deduce that : 


$$
S=\frac{\mu+\gamma}{\beta_{1}+\beta_{2} \delta}
$$

Now from $(1)+(2)$, we deduce that :

$I_{1}=\frac{\Lambda-\mu S}{\mu+d}=\frac{\Lambda\left(\beta_{1}+\beta_{2} \delta\right)-\mu(\mu+\gamma)}{\left.\beta_{1}+\beta_{2} \delta\right)(\mu+d)}$

Let $E^{*}=\left(S^{*}, I_{1}^{*}, I_{2}^{*}\right)$ where $S^{*}=\frac{\mu+\gamma}{\beta_{1}+\beta_{2} \delta}, I_{1}^{*}=\frac{\Lambda-\mu S^{*}}{\mu+d}$ and $I_{2}^{*}=\delta I_{1}^{*}$ where $\delta=\frac{\gamma}{\mu+d}$.

then

- The Jacobian matrix at the endemic equilibrium point $E^{*}=\left(S^{*}, I_{1}^{*}, I_{2}^{*}\right)$ of the system (*) is:

$J_{E^{*}}=\left(\begin{array}{lll}-\beta_{1} I_{1}^{*}-\beta_{2} I_{2}^{*}-\mu & -\beta_{1} S^{*} & -\beta_{2} S^{*} \\ \beta_{1} I_{1}^{*}+\beta_{2} I_{2}^{*} & \beta_{1} S^{*}-\gamma-\mu & \beta_{2} S^{*} \\ 0 & \gamma & -d-\mu\end{array}\right)$

and

- the second additive compound matrix associated to $J_{E^{*}}$ is:

$J_{E^{*}}^{[2]}=\left(\begin{array}{lll}-\beta_{1} I_{1}^{*}-\beta_{2} I_{2}^{*}+\beta_{1} S^{*}-\gamma-2 \mu & \beta_{2} S^{*} & \beta_{2} S^{*} \\ \gamma & -\beta_{1} I_{1}^{*}-\beta_{2} I_{2}^{*}-2 \mu-d & -\beta_{1} S^{*} \\ 0 & \beta_{1} I_{1}^{*}+\beta_{2} I_{2}^{*} & \beta_{1} S^{*}-d-\gamma-2 \mu\end{array}\right)$

\section{Proposition 4.9}

Let

$\bullet_{1} \beta_{2}<\frac{\gamma}{\delta^{2}}$

$\bullet_{2} \frac{(\mu+\gamma)(\mu+d)\left(\beta_{1}+\beta_{2} \delta\right)}{\Lambda\left(\beta_{1}+\beta_{2} \delta\right)-\mu(\mu+\gamma)}+\frac{\beta_{1}(\mu+\gamma)}{\beta_{1}+\beta_{2} \delta}<d+\gamma+2 \mu$

$\bullet_{3} \beta_{2} \delta \Lambda+\mu(\gamma+\mu)<\beta_{1} \Lambda$.

then the endemic equilibrium point of $(*)$ is asymptotically stable.

Proof

Let $\mathbb{P}=\left(\begin{array}{ccc}I_{2}^{*} & 0 & 0 \\ 0 & I_{1}^{*} & 0 \\ 0 & 0 & S^{*}\end{array}\right)$

then the matrix $J_{E^{*}}^{[2]}$ is similar to matrix $\mathbb{A}=\mathbb{P} J_{E^{*}}^{[2]} \mathbb{P}^{-1}=\left(a_{i j}\right)_{1 \leq i, j \leq 3}$ which is given by :

$$
\mathbb{A}=\left(\begin{array}{lcc}
-\beta_{1} I_{1}^{*}-\beta_{2} I_{2}^{*}+\beta_{1} S^{*}-\gamma-2 \mu & \beta_{2} S^{*} \frac{I_{2}^{*}}{I_{1}^{*}} & \beta_{2} S^{*} \frac{I_{2}^{*}}{S^{*}} \\
\gamma \frac{I_{1}^{*}}{I_{2}^{*}} & -\beta_{1} I_{1}^{*}-\beta_{2} I_{2}^{*}-2 \mu-d & -\beta_{1} S^{*} \frac{I_{1}^{*}}{S^{*}} \\
0 & \left(\beta_{1} I_{1}^{*}+\beta_{2} I_{2}^{*}\right) \frac{S^{*}}{I_{1}^{*}} & \beta_{1} S^{*}-d-\gamma-2 \mu
\end{array}\right)
$$

Under the conditions $\bullet_{1}$ and $\bullet_{2}$, we observe that the diagonal elements of $\mathbb{A}$ are negative and;

(1) $a_{11}+\left|a_{12}\right|+\left|a_{13}\right|<0$ 
(2) $a_{22}+\left|a_{21}\right|+\left|a_{23}\right|<0$

(1) $a_{33}+\left|a_{32}\right|<0$

i.e $\mathbb{A}$ is diagonally dominant in rows.

In order to apply the corollary of the Li-Wang criterion, it remains to calculate the determinant of $J_{E^{*}}$

$\operatorname{det}_{E^{*}}=\left|\begin{array}{lll}-\beta_{1} I_{1}^{*}-\beta_{2} I_{2}^{*}-\mu & -\beta_{1} S^{*} & -\beta_{2} S^{*} \\ \beta_{1} I_{1}^{*}+\beta_{2} I_{2}^{*} & \beta_{1} S^{*}-\gamma-\mu & \beta_{2} S^{*} \\ 0 & \gamma & -d-\mu\end{array}\right|$

Under condition $\bullet_{3}$ we deduce that $\operatorname{det}_{E^{*}}<0$

•n=4:A $A^{[2]}=\left(\begin{array}{llllll}a_{11}+a_{22} & a_{23} & a_{24} & -a_{13} & -a_{14} & 0 \\ & & & & & \\ a_{32} & a_{11}+a_{33} & a_{34} & a_{12} & 0 & -a_{14} \\ a_{42} & a_{43} & a_{11}+a_{44} & 0 & a_{12} & a_{13} \\ -a_{13} & a_{21} & 0 & a_{22}+a_{33} & a_{34} & -a_{24} \\ -a_{41} & 0 & a_{21} & a_{43} & a_{22}+a_{44} & a_{23} \\ 0 & -a_{41} & a_{31} & -a_{42} & a_{32} & a_{33}+a_{44}\end{array}\right)$

In next lemma, we give the explicit entries of second additive compound matrix of $n \times n$ matrix $A=\left(a_{i j}\right)$ where $n=5$

\section{Lemma 4.10}

For $n=5$, an explicit expression of second additive compound matrix $A^{[2]}$ is given by:

$$
A^{[2]}=\left(\begin{array}{cccccccccc}
a_{11}+a_{22} & a_{23} & a_{24} & a_{25} & -a_{13} & -a_{14} & -a_{15} & 0 & 0 & 0 \\
a_{32} & a_{11}+a_{33} & a_{34} & a_{35} & a_{12} & 0 & 0 & -a_{14} & -a_{15} & 0 \\
a_{42} & a_{43} & a_{11}+a_{44} & a_{45} & 0 & a_{12} & 0 & a_{13} & 0 & -a_{15} \\
a_{52} & a_{53} & a_{54} & a_{11}+a_{55} & 0 & 0 & a_{12} & 0 & a_{13} & a_{14} \\
-a_{31} & a_{21} & 0 & 0 & a_{22}+a_{33} & a_{34} & a_{35} & -a_{24} & -a_{25} & 0 \\
-a_{41} & 0 & a_{21} & 0 & a_{43} & a_{22}+a_{44} & a_{45} & a_{23} & 0 & -a_{25} \\
-a_{51} & 0 & 0 & a_{21} & a_{53} & a_{45} & a_{22}+a_{55} & 0 & a_{23} & a_{24} \\
-0 & -a_{41} & a_{31} & 0 & -a_{42} & a_{32} & 0 & a_{33}+a_{44} & a_{45} & -a_{35} \\
0 & -a_{51} & 0 & a_{31} & -a_{52} & 0 & a_{32} & a_{54} & a_{33}+a_{55} & a_{34} \\
0 & 0 & -a_{51} & a_{41} & 0 & -a_{52} & a_{42} & -a_{53} & -a_{43} & a_{44}+a_{55}
\end{array}\right)
$$

- As $a_{13}=0, a_{23}=0, a_{24}=0, a_{25}=0, a_{31}=0, a_{35}=0, a_{41}=0, a_{45}=0$ and $a_{54}=0$ in $\mathbb{J}_{p^{*}}^{[2]}$, we deduce that the explicit expression of second additive compound matrix $\mathbb{J}_{p^{*}}^{[2]}$ where $P^{*}=\left(\frac{B}{\mu}, 0,0,0,0\right)$ is : 


$$
\mathbb{J}_{p^{*}}^{[2]}=\left(\begin{array}{llllllllll}
a_{11}+a_{22} & 0 & 0 & 0 & 0 & -a_{14} & -a_{15} & 0 & 0 & 0 \\
a_{32} & a_{11}+a_{33} & a_{34} & a_{35} & a_{12} & 0 & 0 & -a_{14} & -a_{15} & 0 \\
a_{42} & a_{43} & a_{11}+a_{44} & a_{45} & 0 & a_{12} & 0 & 0 & 0 & -a_{15} \\
a_{52} & a_{53} & 0 & a_{11}+a_{55} & 0 & 0 & a_{12} & 0 & 0 & a_{14} \\
0 & a_{21} & 0 & 0 & a_{22}+a_{33} & a_{34} & a_{35} & 0 & -a_{25} & 0 \\
0 & 0 & a_{21} & 0 & a_{43} & a_{22}+a_{44} & r e d 0 & r e d 0 & 0 & 0 \\
-a_{51} & 0 & 0 & a_{21} & a_{53} & r e d 0 & a_{22}+a_{55} & 0 & 0 & 0 \\
0 & -a_{41} & 0 & 0 & -a_{42} & a_{32} & 0 & a_{33}+a_{44} & 0 & 0 \\
0 & -a_{51} & 0 & 0 & -a_{52} & 0 & a_{32} & a_{54} & a_{33}+a_{55} & a_{34} \\
0 & 0 & -a_{51} & 0 & 0 & -a_{52} & a_{42} & -a_{53} & -a_{43} & a_{44}+a_{55}
\end{array}\right)=
$$

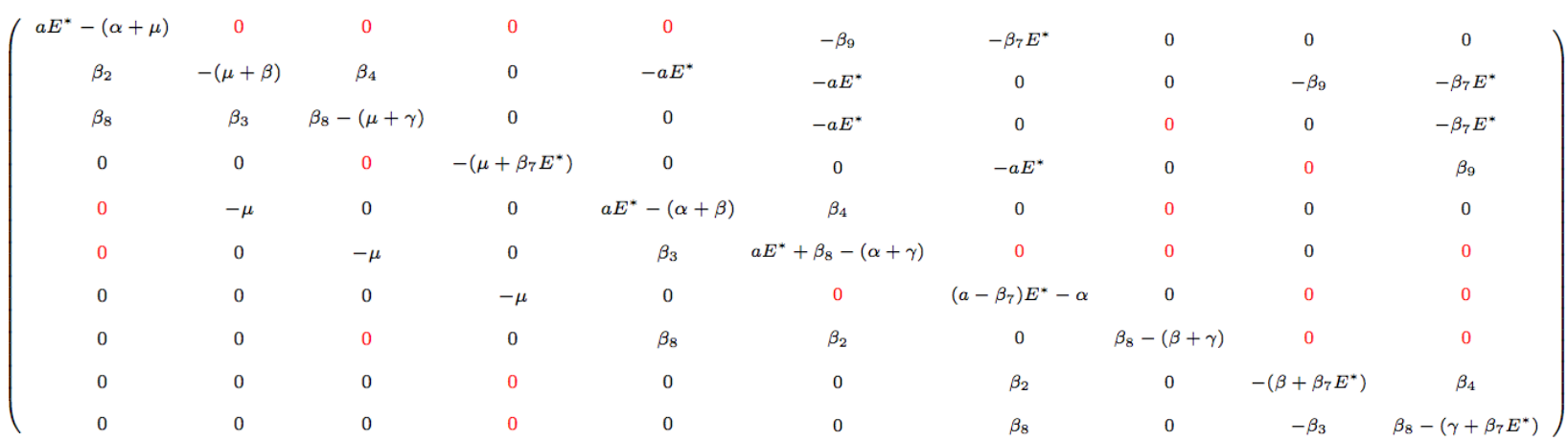

where

$a=\beta_{1}-\beta_{10}, \alpha=\beta_{2}+\beta_{6}+\beta_{8}+\mu, \beta=\beta_{2}+\beta_{5}+\mu$ and $\gamma=\beta_{4}+\beta_{9}+\mu$.

\section{Theorem 4.11}

(i) If $\beta_{10}<\beta_{1}$ and $2 \beta_{8} a E^{*}+\alpha \gamma+\beta_{8} \beta_{9}>\beta_{8} \alpha$ then $\operatorname{det} \mathbb{J}_{p^{0}}<0$.

(ii) if we have :

(a) $\beta_{3}<\beta$

(b) $\frac{2\left(\beta_{1}-\beta_{10}\right) B}{\mu}<\beta_{6}+\mu$

(c) $\beta_{9}<\frac{\beta_{7} B}{\mu}$

(d) $\beta_{8}+\frac{\beta_{7} B}{\mu}<\mu$ then $\mathbb{J}_{p^{0}}^{[2]}$ is diagonally dominant in columns.

(iii) the equilibrium point of (4.1) is asymptotically stable.

\section{Conclusions}

- In this part I, a generalized SEIR model of COVID-19 was discussed. After a glance on basic properties of the model including, the basic reproduction number and the equilibria of the model, we turned on the stability of these states. It was proved that the free equilibrium state is locally as well as globally asymptotically stable when $\mathcal{R}_{0}<$ 1. Furthermore, the second additive compound matrix approach was used to establish the local asymptotic stability of free equilibrium state when $\mathcal{R}_{0}>1$. 
- In second paper (Part II), In order to control the Covid-19 system, i.e., force the trajectories to go to the equilibria we will add some control parameters with uncertain parameters to stabilize the five-dimensional Covid-19 system studied in this paper.

Based on compound matrices theory, we have constructed in Intissar (2020) [3] the controllers:

$\mathbb{U}=\left(\begin{array}{ccccc}0 & u_{1} & 0 & 0 & 0 \\ u_{2} & 0 & 0 & 0 & 0 \\ 0 & 0 & 0 & 0 & 0 \\ 0 & 0 & 0 & 0 & 0 \\ u_{3} & 0 & 0 & 0 & 0\end{array}\right)$ i.e. $\mathbb{U}=\left(u_{i j}\right)$ where $u_{i j}=0$ except $\left(u_{12}, u_{21}, u_{51}\right) \in \mathbb{R}^{3} ; 1 \leq i, j \leq 5$

to stabilize the system (4.1), in particular to study the stability of following matrix :

$\mathbb{J}_{p^{*}, u_{1}, u_{2}, u_{3}}=\mathbb{J}_{p^{*}}+\mathbb{U}$ and its second additive compound matrix $\left(\mathbb{J}_{p^{*}}+\mathbb{U}\right)^{[2]}$, by applying again the criterion of LiWang on second compound matrix associated to the system (4.1) with these controllers.

We have constructed a Lyapunov function $\mathbb{L}$ of the system (4.1) for apply the classical Lyapunov theorem and to get:

\section{Theorem 4.12}

(i) $(0,0,0,0,0)$ is a stable equilibrium point in the sense of Lyapunov.

(ii) $\mathbb{V}(e, i, c, h, d)<0,0<\|(e, i, c, h, d)\|<r_{1}$ for some $r_{1}$, i.e. if $\mathbb{L}$ is lnd.

(iii) $(0,0,0,0,0)$ is an asymptotically stable equilibrium point.

\section{Acknowledgements}

I thank my soon Jean-Karim Intissar for several important suggestions and comments on this work.

\section{Declaration of Competing Interest}

The authors declare that they have no known competing financial interests or personal relationships that could have appeared to influence the work reported in this paper.

\section{Ethical Approval}

The manuscript does not contain experiments on animals and humans; hence ethical permission not required.

\section{References}

[1] Shah, N. H., Suthar, A. H., \& Jayswal, E. N. (2020). Control Strategies to Curtail Transmission of COVID-19. doi:10.1101/2020.04.04.20053173.

[2] Li, M. Y., \& Wang, L. (1998). A Criterion for Stability of Matrices. Journal of Mathematical Analysis and Applications, 225(1), 249-264. doi:10.1006/jmaa.1998.6020.

[3] Intissar, A. (2020). Construction of controllers to stabilize five-dimensional Covid-19 mathematical system with uncertain parameters (Preprint).

[4] Arnold, V. I. (2012). Geometrical methods in the theory of ordinary differential equations (Vol. 250). Springer Science \& Business Media.

[5] Banks, J., Brooks, J., Cairns, G., Davis, G., \& Stacey, P. (1992). On Devaney's definition of chaos. The American mathematical monthly, 99(4), 332-334. doi:10.1080/00029890.1992.11995856.

[6] Heffernan, J., Smith, R. ., \& Wahl, L. . (2005). Perspectives on the basic reproductive ratio. Journal of The Royal Society Interface, 2(4), 281-293. doi:10.1098/rsif.2005.0042.

[7] Diekmann, O., Heesterbeek, J. A. P., \& Metz, J. A. J. (1990). On the definition and the computation of the basic reproduction ratio $\mathrm{R} 0$ in models for infectious diseases in heterogeneous populations. Journal of Mathematical Biology, 28(4). doi:10.1007/bf00178324.

[8] Van den Driessche, P., \& Watmough, J. (2002). Reproduction numbers and sub-threshold endemic equilibria for compartmental models of disease transmission. Mathematical Biosciences, 180(1-2), 29-48. doi:10.1016/s0025-5564(02)00108-6. 
[9] Li, M. Y., Graef, J. R., Wang, L., \& Karsai, J. (1999). Global dynamics of a SEIR model with varying total population size. Mathematical Biosciences, 160(2), 191-213. doi:10.1016/s0025-5564(99)00030-9.

[10] Beretta, E., \& Capasso, V. (1986). On the general structure of epidemic systems. Global asymptotic stability. Computers \& Mathematics with Applications, 12(6), 677-694. doi:10.1016/0898-1221(86)90054-4.

[11] Sun, C., \& Hsieh, Y.-H. (2010). Global analysis of an SEIR model with varying population size and vaccination. Applied Mathematical Modelling, 34(10), 2685-2697. doi:10.1016/j.apm.2009.12.005.

[12] Poincaré, H. (1890). Sur le problème des trois corps et les équations de la dynamique. Acta Mathematica, 13(1), A3-A270.

[13] Grobman, D. M. (1959). Homeomorphism of systems of differential equations. Doklady Akademii Nauk SSSR, 128(5), 880881.

[14] Hartman, P. (1960). A lemma in the theory of structural stability of differential equations. Proceedings of the American Mathematical Society, 11(4), 610-620. doi:10.2307/2034720.

[15] Hartman, P. (1964). Ordinary Differential Equations, Wiley, New York.

[16] Perko, L. (2013). Differential equations and dynamical systems (Vol. 7). Springer Science \& Business Media.

[17] Wang, X. (2020). A Global Hartman-Grobman Theorem. arXiv preprint arXiv:2002.06094.

[18] Fiedler, M. (1974). Additive compound matrices and an inequality for eigenvalues of symmetric stochastic matrices. Czechoslovak Mathematical Journal, 24(3), 392-402.

[19] Muldowney, J. (1990). Compound Matrices And Ordinary Differential Equations. The Rocky Mountain Journal of Mathematics, 20(4), 857-872.

[20] Intissa, A. and Intissa, J-K (2017). Differential Calculus, Principles and Applications, CEPADUES Editions, I.S.B.N: 9782364935884 .

[21] Aimar, M-T, Intissar A. and Intissar, J-K (2019). Elementary Mathematical Analysis of Chaos: in Classical and a few New Dynamical Systems From Lorenz to Gribov-Intissar systems, Kindle Edition, ASIN: B07QV7JPLW.

[22] Khanh, N. H. (2016). Stability analysis of an influenza virus model with disease resistance. Journal of the Egyptian Mathematical Society, 24(2), 193-199. doi:10.1016/j.joems.2015.02.003.

[23] Coppel, W. A. (1965). Stability and asymptotic behavior of differential equations (DC Heath \& Co., Boston, MA).

[24] Bylov, B. F., Vinograd, R. E., Grobman, D. M., \& Nemytski1, V. V. (1966). Lyapunov exponents theory and its applications to stability problems. Nauka, Moscow.

[25] Lozinskii, S. M. (1958). Error estimate for numerical integration of ordinary differential equations. I. Izvestiya Vysshikh Uchebnykh Zavedenii. Matematika, (5), 52-90.

[26] Dahlquist, G. (1958). Stability and error bounds in the numerical integration of ordinary differential equations (Doctoral dissertation, Almqvist \& Wiksell).

[27] Daletskii, Yu. L. and Krein, M. G. (1970). Stability of Solutions of Differential Equations in Banach Space, in Nonlinear Analysis and Its Applications (Nauka, Moscow).

[28] Perov, A. I. (2002). New stability criteria for constant-coefficient linear systems. Automation and Remote Control, 63(2), 189199. doi:10.1023/A:1014287306173.

[29] Price, G. B. (1951). Bounds for determinants with dominant principal diagonal. Proceedings of the American Mathematical Society, 2(3), 497-502. doi:10.2307/2031782.

[30] Ostrowski, A. (1937). Über die determinanten mit uberwiegender Hauptdiagonale. Commentarii Mathematici Helvetici, 10(1), 69-96. doi:10.1007/bf01214284.

[31] Ostrowski, A. (1952). Note on Bounds for Determinants with Dominant Principal Diagonal. Proceedings of the American Mathematical Society, 3(1), 26-30. doi:10.2307/2032450.

[32] Ostrowski, A. M. (1955). Note on bounds for some determinants. Duke mathematical journal, 22(1), 95-102. doi:10.1215/S0012-7094-55-02208-0.

[33] Brenner, J. (1954). A Bound for a Determinant with Dominant Main Diagonal. Proceedings of the American Mathematical Society, 5(4), 631-634. doi:10.2307/2032049.

[34] Brenner, J. (1957). Bounds for Determinants. II. Proceedings of the American Mathematical Society, 8(3), 532-534. doi:10.2307/2033510. 
[35] Schneider, H. (1953). An Inequality for Latent Roots Applied To Determinants with Dominant Principal Diagonal. Journal of the London Mathematical Society, s1-28(1), 8-20. doi:10.1112/jlms/s1-28.1.8.

[36] Marshall, A. W., Olkin, I., \& Arnold, B. C. (2011). Inequalities: Theory of Majorization and Its Applications. Springer Series in Statistics. doi:10.1007/978-0-387-68276-1.

[37] Aitken, A. C. (2017). Determinants and matrices. Oliver \& Boyd, Edinburgh.

[38] Marcus, M. (1973-1975). Finite Dimensional Multilinear Algebra, Two Volumes. Marcel Dekker, New York.

[39] Mitrouli, M., \& Koukouvinos, C. (1997). On the computation of the Smith normal form of compound matrices. Numerical Algorithms, 16(2), 95-105. doi:10.1023/A:1019182912676.

[40] Kravvaritis, C., \& Mitrouli, M. (2008). Compound matrices: properties, numerical issues and analytical computations. Numerical Algorithms, 50(2), 155-177. doi:10.1007/s11075-008-9222-7.

[41] Elsner, L., Hershkowitz, D., \& Schneider, H. (2000). Bounds on Norms of Compound Matrices and on Products of Eigenvalues. Bulletin of the London Mathematical Society, 32(1), 15-24. doi:10.1112/s0024609399006402.

[42] Johnson, C. R. (1970). Positive Definite Matrices. The American Mathematical Monthly, 77(3), $259-264$. doi:10.1080/00029890.1970.11992465.

[43] Pease, M. C. (1965). Methods of matrix algebra (No. 512.896 P43).

[44] Marcus, M., \& Minc, H. (1988). Introduction to linear algebra. Courier Corporation.

[45] Marcus, M. and Minc, H. (1992). Positive Definite Matrices. § 4.12 in A Survey of Matrix Theory and Matrix Inequalities. New York: Dover, p. 69.

[46] Golub, G. H. and Van Loan, C. F. (1996). Positive Definite Systems. § 4.2 in Matrix Computations, 3rd ed. Baltimore, MD: Johns Hopkins University Press, pp. 140-141.

[47] Fiedler, M., \& Ptak, V. (1962). On matrices with non-positive off-diagonal elements and positive principal minors. Czechoslovak Mathematical Journal, 12(3), 382-400.

[48] Liao, X. (2002). On asympotic behavior of solutions to several classes of discrete dynamical systems. Science in China Series A: Mathematics, 45(4), 432-442. doi:10.1007/bf02872331.

[49] Zhang, Y., \& Zheng, B. (2013). The Stability Criteria with Compound Matrices. Abstract and Applied Analysis, 2013 , 1-5. doi:10.1155/2013/930576.

[50] Van den Driessche, P., \& Watmough, J. (2008). Further Notes on the Basic Reproduction Number. Lecture Notes in Mathematics, 159-178. doi:10.1007/978-3-540-78911-6_6.

[51] Intissar, A. (2019). Elementary Proof of a generalization of the Perron-Frobenius theorem in an ordered Banach space. arXiv preprint arXiv: 1910.01120.

[52] Berman, A. and Plemmons, R. J. Nonnegative Matrices in the Mathematical Sciences, Academic Press, New York, (1979), chapter 6 .

[53] Horn, R. A., Horn, R. A., \& Johnson, C. R. (1994). Topics in matrix analysis. Cambridge university press.

[54] Hawkins, D., \& Simon, H. (1949). Note: Some Conditions of Macroeconomic Stability. Econometrica, 17(3/4), 245-248. doi: $10.2307 / 1905526$.

[55] Fan, K. (1958). Topological proofs for certain theorems on matrices with non-negative elements. Monatshefte Fur Mathematik, 62(3), 219-237. doi:10.1007/bf01303967.

[56] Cheng, Z. J., \& Shan, J. (2019). Novel coronavirus: where we are and what we know. Infection, 48 (2): 155-63.

[57] Nickalls, R. (1993). A New Approach to Solving the Cubic: Cardan's Solution Revealed. The Mathematical Gazette, 77(480), 354-359. doi:10.2307/3619777. 